ARTICLE

\title{
Ribosome-bound Get4/5 facilitates the capture of tail-anchored proteins by Sgt2 in yeast
}

Ying Zhang (1) 1,2,6, Evelina De Laurentiis ${ }^{3,4,6}$, Katherine E. Bohnsack (10 ${ }^{3}$, Mascha Wahlig ${ }^{1,2}$, Namit Ranjan (10 5, Simon Gruseck ${ }^{1,2}$, Philipp Hackert ${ }^{3}$, Tina Wölfle ${ }^{1,2}$, Marina V. Rodnina (1) ${ }^{4,5}$, Blanche Schwappach (1) ${ }^{3,4 凶} \&$ Sabine Rospert (10) $1,2 \otimes$

The guided entry of tail-anchored proteins (GET) pathway assists in the posttranslational delivery of tail-anchored proteins, containing a single C-terminal transmembrane domain, to the ER. Here we uncover how the yeast GET pathway component Get4/5 facilitates capture of tail-anchored proteins by Sgt2, which interacts with tail-anchors and hands them over to the targeting component Get3. Get4/5 binds directly and with high affinity to ribosomes, positions Sgt2 close to the ribosomal tunnel exit, and facilitates the capture of tail-anchored proteins by Sgt2. The contact sites of Get4/5 on the ribosome overlap with those of SRP, the factor mediating cotranslational ER-targeting. Exposure of internal transmembrane domains at the tunnel exit induces high-affinity ribosome binding of SRP, which in turn prevents ribosome binding of Get4/5. In this way, the position of a transmembrane domain within nascent ER-targeted proteins mediates partitioning into either the GET or SRP pathway directly at the ribosomal tunnel exit.

\footnotetext{
${ }^{1}$ Institute of Biochemistry and Molecular Biology, University of Freiburg, Freiburg, Germany. ${ }^{2}$ BIOSS Centre for Biological Signaling Studies, University of Freiburg, Freiburg, Germany. ${ }^{3}$ Department of Molecular Biology, University Medical Center Göttingen, Göttingen, Germany. ${ }^{4}$ Cluster of Excellence "Multiscale Bioimaging: from Molecular Machines to Networks of Excitable Cells" (MBExC), Göttingen, Germany. ${ }^{5}$ Max-Planck Institute for Biophysical Chemistry, Göttingen, Germany. ${ }^{9}$ These authors contributed equally: Ying Zhang, Evelina De Laurentiis. ${ }^{\circledR}$ email: blanche.schwappach@med.uni-goettingen.de; sabine.rospert@biochemie.uni-freiburg.de
} 
ntegral membrane proteins that follow the secretory and endocytic pathways are synthesized on cytosolic ribosomes and targeted to the membrane of the endoplasmic reticulum (ER). A general challenge during the targeting of membrane proteins is aggregation of hydrophobic transmembrane (TM) domains prior to insertion into the lipid bilayer. In the case of ER-targeted proteins, aggregation is prevented by sophisticated machineries, which selectively recognize and shield TM domains in the cytosol ${ }^{1-5}$.

Many ER membrane proteins are targeted cotranslationally with the assistance of the highly conserved signal recognition particle (SRP) ${ }^{1-5}$. Eukaryotic SRP is a multi-subunit ribonucleoprotein complex, which binds to translating ribosomes exposing nascent SRP recognition sequences comprising $\mathrm{N}$-terminal signal sequences and signal anchor (SA) sequences. The latter initially serve as targeting signals and ultimately as TM domains that anchor mature ER-targeted proteins within the membrane. Ribosome-bound SRP is positioned such that its $54 \mathrm{kDa}$ subunit termed Srp54 binds to emerging SRP recognition sequences in close proximity to the polypeptide exit tunnel of the ribosome. The complex consisting of SRP and the ribosome-bound nascent chain (RNC) is subsequently targeted to the ER membrane where SRP interacts with the SRP receptor, resulting in SRP release and docking of the translating ribosome to the ER translocon. As translation proceeds, the nascent membrane protein is inserted into the ER membrane ${ }^{1-5}$.

The SRP-dependent mode of targeting elegantly circumvents aggregation of TM proteins, as it couples protein synthesis with membrane insertion of TM domains. However, a specific subgroup of TM proteins cannot enter the cotranslational, SRPdependent pathway, because these proteins possess only a single TM domain at their very C-terminus, termed a tail-anchor (TA) sequence. TA sequences become exposed to the outside of the polypeptide exit tunnel only after the nascent polypeptide is released from the ribosome upon translation termination. The pathway responsible for targeting of TA proteins to the ER membrane is termed the guided entry of TA protein (GET) pathway $^{6-9}$. According to current models, the GET pathway is initiated shortly after release of TA proteins from the ribosome, when the hydrophobic TA sequence is captured by the C-terminal domain of a homodimeric chaperone-like protein termed Sgt $2^{10,11}$. Within the Sgt2/TA protein complex, the $\mathrm{N}$ terminal domain of Sgt 2 recruits the Get5 subunit of the heterotetrameric Get $4{ }_{2} \mathrm{Get} 5{ }_{2}$ complex (Get4/5), allowing, in turn, binding of the homodimeric targeting factor Get3 to Get $4^{10-13}$. After the TA sequence is transferred from Sgt 2 to Get 3 and the Get3/TA protein complex dissociates from the pre-targeting complex, the TA protein is delivered to the Get $1 / 2$ insertase in the ER membrane $e^{1,5,11,14-16}$.

Considering the relatively recent discovery of the GET pathway, it is remarkable how well many of its features are understood at structural and mechanistic levels, including TA protein capture by Get3 and subsequent membrane delivery $1,5,11,14-17$. However, our understanding of the initial phase of the GET pathway lags behind. SRP scans translating ribosomes and displays enhanced binding affinity even before nascent TM domains become fully exposed outside of the exit tunnel ${ }^{4,18-22}$. In contrast, precisely where and when Sgt 2 captures TA proteins and why Get 3 does not bind directly to released TA proteins is currently not understood. This may be due to the immense experimental challenge of analyzing the short period between peptidyl-tRNA cleavage upon translation termination and complete exit of released polypeptides from the ribosomal tunnel.

Preliminary evidence suggests that, even though the GET pathway is considered to employ a posttranslational mechanism, it may be directly coupled to the emergence of its clients from the ribosome. A high-throughput study identified Get5 as a ribosome-associated protein ${ }^{23}$ and later work revealed that indeed the yeast Get4/5 complex binds to ribosomes independently of their translational status, however, displays enhanced affinity to those ribosomes with a TM sequence inside the ribosomal tunnel ${ }^{24}$. These findings hint at a direct transfer of TA proteins from the ribosome to the GET pathway, with Get4/5 potentially being the earliest sensing component. Such a scenario, however, is difficult to reconcile with current models, which appear well supported by extensive in vitro reconstitution approaches and consider Sgt 2 as the first GET pathway component interacting with TA proteins ${ }^{1,5,11,14-16}$.

Here we investigated the early steps of the GET pathway, with a focus on the steps that occur upon exit of TA sequences from the polypeptide exit tunnel of the ribosome. We show that Get4/5 binds to ribosomes with high affinity via the Get5 subunit, which contacts ribosomal proteins Rpl26/uL24 and Rpl35/uL29 at the exit of the ribosomal tunnel (names of ribosomal proteins are given according to the yeast standard nomenclature). Get4 is not required for stable ribosome-binding of Get5, but binds near h46/ h47 of the $25 \mathrm{~S}$ rRNA in the complex. The ribosomal binding site of Get5 overlaps with that of SRP, and SRP can prevent Get4/5 from binding to the ribosome. However, once Get $4 / 5$ is bound to the ribosome, Sgt 2 is recruited to the tunnel exit and gains direct access to exposed TA sequences. This Get4/5-dependent recruitment of Sgt 2 improves the efficiency by which Sgt2 captures TA proteins upon release from the ribosome. The combined data indicate that ribosome-bound Get $4 / 5$ couples translation of TA proteins to their entry into the GET pathway, thereby minimizing cytosolic exposure of TA sequences.

\section{Results}

The Get4/5 complex interacts with ribosomes via Get5. The cytosolic Get $4 / 5$ complex is distributed between a free and a ribosome-bound pool (Fig. 1a, b, and ref. ${ }^{24}$ ). To understand whether one or both subunits of Get $4 / 5$ are required for ribosome binding, recruitment of Get4 to the ribosome was analyzed in a $\Delta$ get5 strain, and Get5 was analyzed in a $\Delta$ get 4 strain. As Get5 is unstable in a $\Delta$ get4 strain (Supplementary Fig. 1), Get5 was overexpressed in the $\Delta$ get 4 background (Fig. 1a, $\Delta$ get $4+$ Get $5 \uparrow$ ). Ribosome binding was assessed in a sedimentation assay by separating ribosomes from cytosolic components under low salt (120 mM KOAc) or high salt $(800 \mathrm{mM} \mathrm{KOAc})$ conditions. In this assay, ribosome-bound factors cosediment with ribosomes under low-salt conditions, but are released to the cytosolic supernatant when the salt concentration is high. The assay was employed to distinguish ribosome association from protein aggregation, because protein aggregates cosediment with core ribosomal particles under low as well as high salt conditions ${ }^{25}$. In the wild type, $>40 \%$ of Get $4 / 5$ was ribosome-bound under low salt conditions and was released from the ribosome under high salt conditions (Fig. 1a, b, wild type). When Get 4 was absent, $>70 \%$ of overexpressed Get5 was ribosome-bound in a salt-sensitive manner (Fig. 1a, b, $\Delta$ get $4+$ Get $5 \uparrow$ ). When Get5 was absent, a significant amount of Get4 was recovered in the pellet even under high salt conditions, suggesting partial aggregation of Get4 (Fig. 1a, white asterisk). The fraction of Get4, which was recovered in the ribosomal pellet in a salt-sensitive manner in the absence of Get5 was strongly reduced to $\sim 7 \%$ (Fig. 1a, b, $\Delta$ get 5 ). We conclude that Get5 interacts with ribosomes stably and independently of Get4, whereas Get 4 by itself associates with ribosomes with much lower affinity and requires Get5 for efficient ribosome binding.

Get4/5 binds to non-translating ribosomes with high affinity. In order to determine the affinity of Get $4 / 5$ to $80 \mathrm{~S}$ ribosomes, the 
a

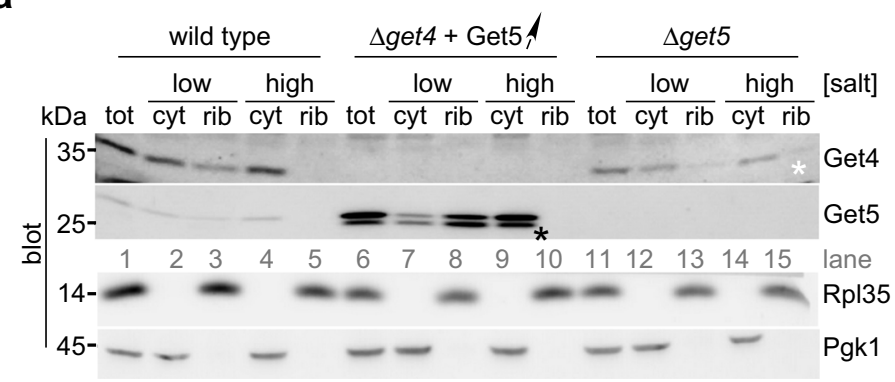

b

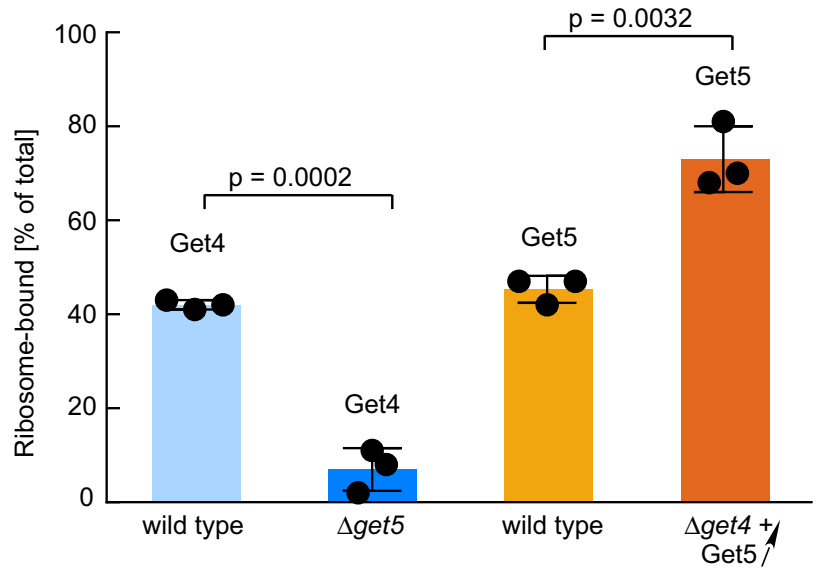

C
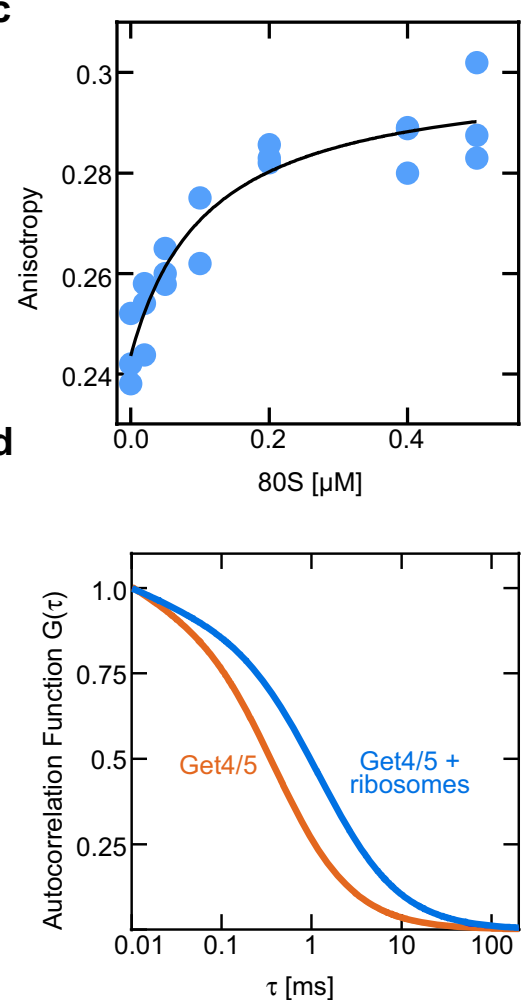

Fig. 1 The Get4/5 complex binds to ribosomes with high affinity. a The Get4/5 complex binds to ribosomes via Get5. Total cell extracts (tot) of wild type, a $\Delta$ get4 strain overexpressing Get5 ( $\Delta$ get4 + Get5 $\uparrow$, Supplementary Fig. 1), or a $\Delta$ get5 strain were separated into a cytosolic fraction (cyt) and a ribosomal pellet (rib) under low salt (120 mM KOAc) or high salt ( $800 \mathrm{mM} \mathrm{KOAc)}$ conditions. The black asterisk indicates the fraction of overexpressed Get5 migrating with lower molecular mass, likely due to partial degradation of overexpressed Get5. The white asterisk indicates the fraction of aggregated Get4, which sediments together with ribosomes under high salt conditions in the absence of Get5. Aliquots were analyzed via immunoblotting with $\alpha$-Get4, $\alpha$-Get5, $\alpha$-Rpl35 (ribosomal marker), and $\alpha$-Pgk1 (cytosolic marker). b Quantification of the fraction of Get4 and Get5 bound to ribosomes. Given is the fraction of Get4 or Get5 recovered in low salt ribosomal pellets (rib) compared with the total (rib+cyt) based on the analysis of experiments as shown in $\mathbf{a}$. Get4 or Get5 recovered in high salt ribosomal pellets was subtracted as a background. The results of each biological replicate are shown as black dots. Error bars indicate the standard deviation of the mean. Indicated $p$ values were calculated using a two-sided Student's $t$ test with GraphPad Prism. c Get4/5 binds to purified $80 \mathrm{~S}$ ribosomes with high affinity. Fluorescence anisotropy-based binding assays were performed with Get4/5-Atto390 (20 nM) and purified $80 \mathrm{~S}$ ribosomes (see Methods). The data were fit to a one site, saturation binding curve, from which the equilibrium dissociation constant and the standard error of the mean $\left(K_{d}=110 \pm 40 \mathrm{nM}\right)$ was determined using GraphPad Prism. $\mathbf{d}$ Get4/5 binding to the 80S ribosome monitored by FCS. Autocorrelation functions are represented for Get4/5-Atto655 alone (red) and in complex with the 80S ribosomes (blue). Concentrations of Get4/5Atto655 and $80 \mathrm{~S}$ ribosomes were $5 \mathrm{nM}$ and $1 \mu \mathrm{M}$, respectively.

Get4/5 complex was purified, Get4 was labeled with a fluorescent dye Atto390 (Get4/5-Atto390, see Methods) and the binding was monitored using the change of the anisotropy of the fluorophore. Fluorescence anisotropy changes followed a saturation binding curve reflecting a single binding site with a $K_{\mathrm{d}}$ of $110 \pm 40 \mathrm{nM}$ (Fig. 1c). To monitor the interaction between Get $4 / 5$ and the ribosome directly, single molecule fluorescence correlation spectroscopy (FCS) was used (Fig. 1d and Supplementary Table 1). FCS reveals the diffusion times of molecules as they move through the confocal volume of a microscope. As the $80 \mathrm{~S}$ ribosome $\left(3.2 \mathrm{MDa}^{26}\right)$ is much larger than Get $4 / 5\left(120 \mathrm{kDa}, \alpha_{2} \beta_{2}\right.$ complex), the diffusion time of Get $4 / 5$ alone will be much faster than when it is in complex with the ribosome. For FCS experiments, Get $4 / 5$ was labeled with Atto655 and the diffusion properties of Get4/5-Atto655 were studied with or without ribosomes. Fitting the FCS autocorrelation functions showed that in the sample with Get4/5-Atto655 alone, the majority of the molecules moved with an average diffusion time of $0.39 \mathrm{~ms}$; a small fraction of molecules that diffused more rapidly likely represented residual free dye (Supplementary Table 1). From the diffusion time of Get4/5-Atto655, we calculated a diffusion coefficient of $87 \pm 2$ $\mu \mathrm{m}^{2} \mathrm{~s}^{-1}$, which is close to the value of $70 \mu \mathrm{m}^{2} \mathrm{~s}^{-1}$ estimated based on the size ${ }^{27}$ and hydrodynamic radius ${ }^{28}$ of Get $4 / 5$. Addition of $80 \mathrm{~S}$ ribosomes changed the diffusion time dramatically to approximately $1.6 \mathrm{~ms}$ for $67 \%$ of Get $4 / 5$-Atto655 molecules, reflecting Get $4 / 5 / 80$ S complex formation. From the diffusion time, we calculated a diffusion coefficient of $20 \mu \mathrm{m}^{2} \mathrm{~s}^{-1}$, which is close to that expected for diffusion of the $80 \mathrm{~S}$ ribosome $\left(12-18 \mu \mathrm{m}^{2} \mathrm{~s}^{-1}\right)^{29,30}$. Together, the data confirm that Get $4 / 5$ binds to ribosomes directly and indicate that the affinity of Get $4 / 5$ for empty ribosomes is in the submicromolar range (see also Supplementary Note 1).

Get5 contacts Rpl35 and Rpl26 at the ribosomal tunnel exit. We next focused on the identification of contacts between Get5 and ribosomal proteins using a crosslinking approach (Fig. 2a). To enhance the occupancy of ribosomes with Get $4 / 5$ during crosslinking, we overexpressed Get4 and an $\mathrm{N}$-terminally $\mathrm{His}_{6}{ }^{-}$ tagged Get5 variant $\left(\mathrm{His}_{6} \mathrm{Get} 5\right)$, or, as a control, Get4 and untagged Get5. Ribosomes from these strains were isolated under low salt conditions, crosslinking was performed, and $\mathrm{His}_{6} \mathrm{Get} 5$ crosslink products were purified under denaturing conditions via Ni-NTA (Fig. 2a and Supplementary Fig. 2a). Analysis of the NiNTA-purified material revealed several $\mathrm{His}_{6} \mathrm{Get} 5$ crosslink 
a

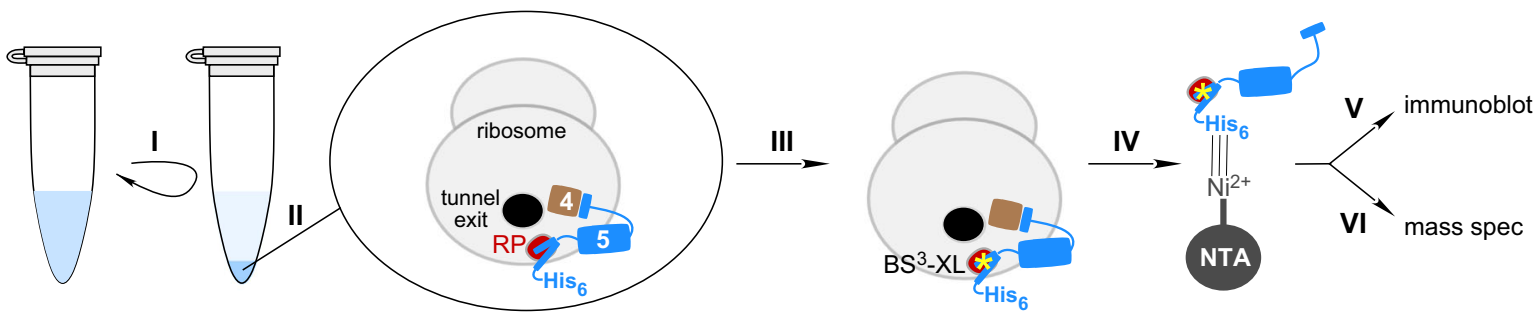

b

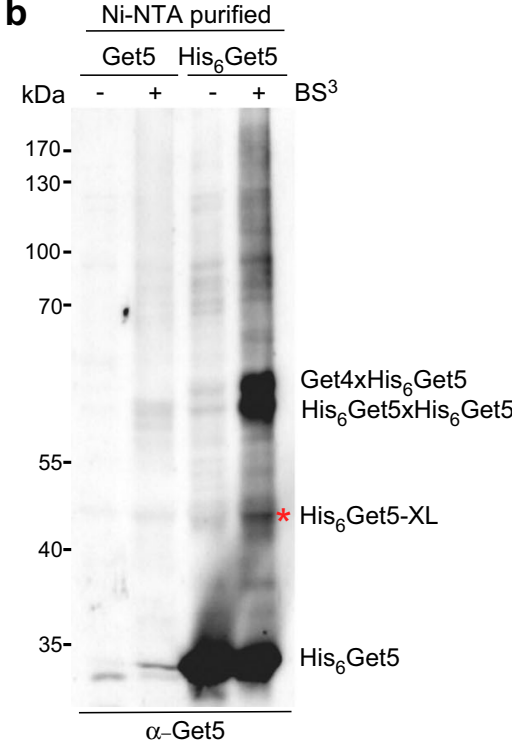

C

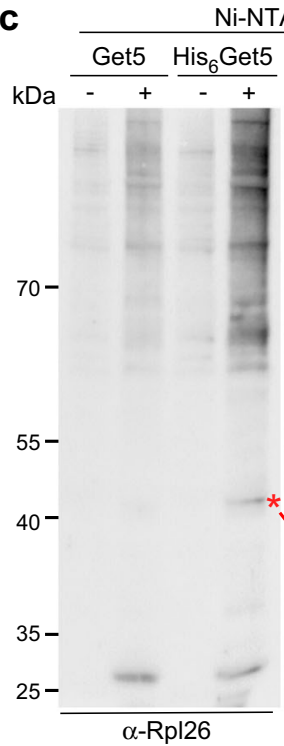

Ni-NTA purified

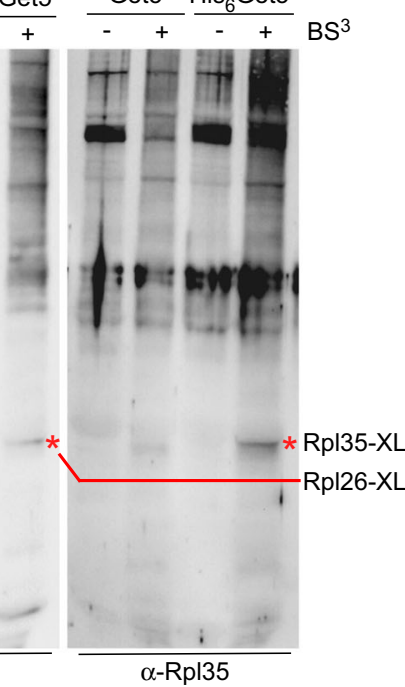

d Ni-NTA purified

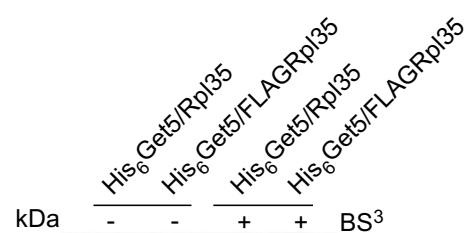

$\mathrm{kDa}$

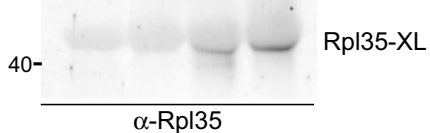

$40-$

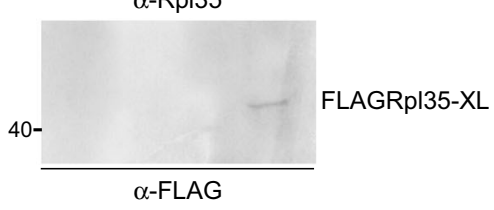

Fig. 2 Ribosome-bound Get5 contacts Rpl26 and Rpl35 at the ribosomal tunnel exit. a Crosslinking analysis of ribosomal protein contacts. Ribosomes in total cell extract were (I) collected via centrifugation, (II) resuspended in low salt buffer, and (III) crosslinked with the homobifunctional, amino reactive crosslinker BS ${ }^{3}$. (IV) The crosslinked material was denatured and subsequently His 6 Get5-containing crosslink products were isolated via Ni-NTA purification (see Methods). His 6 Get5 and crosslinked proteins, termed Ni-NTA purified His ${ }_{6}$ Get5, was analyzed (V) via immunoblotting with antibodies recognizing candidate ribosomal proteins and $(\mathrm{VI})$ via mass spectrometry (see Supplementary Fig. 2). For simplicity, only one heterodimeric subcomplex of the Get $4{ }_{2}$ Get5 5 tetramer is depicted in all cartoons. b, c Immunodetection of Get5 crosslink products. His 6 Get5, with or without crosslinking, was purified via Ni-NTA as described in a from cells expressing either $\mathrm{His}_{6} \mathrm{Get5}$, or, as a control, untagged Get5. Aliquots of the indicated samples were analyzed via immunoblotting with $\alpha$-Get5 (b), $\alpha$-Rpl26, or $\alpha$-Rpl35 (c). Red asterisks indicate crosslink products, recognized by $\alpha$-Get5 (His ${ }_{6} \mathrm{Get} 5-\mathrm{XL}$ in $\mathbf{b}$ ), $\alpha$-Rpl26, or $\alpha$-Rpl35 (Rpl26-XL, Rpl35-XL in c). Crosslink products between His 6 Get5 and Get4 subunits are indicated (see also Supplementary Fig. 2b). d Identification of Rpl35 as a crosslink partner of Get5. Ni-NTA purified His ${ }_{6}$ Get5 was generated as described in panel a from cells coexpressing His 6 Get5 and FLAGtagged Rpl35 (FLAGRpl35). Aliquots were analyzed via immunoblotting with $\alpha$-Rpl35 (upper panel) or $\alpha$-FLAG (lower panel).

products in the low and high molecular mass ranges (Fig. 2b). The most prominent crosslink products comprised the complex of two Get5 moieties (His ${ }_{6}$ Get $_{5} \mathrm{xHis}_{6} \mathrm{Get} 5$ ) and the complex of Get4 and Get5 (Get4xHis ${ }_{6}$ Get5) (Fig. 2b and Supplementary Fig. 2b). The same approach was used for cells overexpressing His $_{6}$-tagged Get4 (His 6 Get4) and untagged Get5; however, no additional crosslink products were detected besides the $\mathrm{His}_{6}$ Get4xGet5 product (Supplementary Fig. 2b). His ${ }_{6}$ Get5 crosslink products were initially probed with antibodies from our collection (a-RPP0/uL10, a-Asc1/RACK1, a-Rps3/uS3, a-Rps9/uS4, aRps20/uS10, a-Rpl4/uL4, a-Rpl17/uL22, a-Rpl31/eL31, a-Rpl25/ uL23, $\alpha$-Rpl16/uL13, $\alpha$-Rpl26/uL24, and $\alpha$-Rpl35/uL29, see Source Data file). This analysis revealed that $\alpha$-Rpl26 and $\alpha$-Rpl35 recognized a $\mathrm{His}_{6} \mathrm{Get} 5$ crosslink product of approximately 40 $\mathrm{kDa}$, which corresponds to the expected mass of Rpl26xHis $\mathrm{Get}_{6}$ or Rpl35xHis ${ }_{6}$ Get5, respectively (Get5: $25 \mathrm{kDa}$, Rpl26: $14 \mathrm{kDa}$, Rpl35: $14 \mathrm{kDa}$ ) (Fig. 2c, Rpl35-XL and Rpl26-XL).

In a parallel approach, crosslinked, Ni-NTA purified $\mathrm{His}_{6} \mathrm{Get} 5$ and appropriate controls (Supplementary Fig. 2c) were analyzed by mass spectrometry (see Methods, Supplementary Dataset 1, and Source Data file). From the data obtained by mass spectrometry, potential ribosomal crosslinking partners of His $_{6}$ Get5 were selected based on the following criteria: (i) the analysis was confined to core ribosomal proteins (RPs); (ii) only those RPs were selected for which the total spectral counts of $\mathrm{His}_{6} \mathrm{Get} 5 \mathrm{xRP}$ was at least 1.4-fold increased compared to the Get5xRP control; and (iii) the $p$ value was $\leq 0.01$ (Supplementary Dataset 1). Only four large subunit ribosomal proteins (Rpl20/ eL20, Rpl23/uL14, Rpl26/uL24, and Rpl37/eL37) and two small subunit ribosomal proteins (Rps19/eS19 and Rps29/uS14) met these criteria (Table 1). The mass spectrometry approach confirmed Rpl26 as a contact of ribosome-bound $\mathrm{His}_{6} \mathrm{Get} 5$, however, did not identify Rpl35 as a crosslinking partner of His $_{6}$ Get5. We therefore tested whether the crosslinked species recognized by $\alpha$-Rpl35 (Fig. 2c) was indeed $\mathrm{His}_{6}$ Get5xRpl35. To that end, we employed strains expressing FLAG-tagged Rpl35 (FLAGRpl35) or endogenous untagged Rpl35 in a His 6 Get5 background. After crosslinking and purification a $\sim 40 \mathrm{kDa}$ crosslink product was detected by both $\alpha$-Rpl35 and $\alpha$-FLAG specifically in the $\mathrm{His}_{6}$ Get5/FLAGRpl35 strain (Fig. 2d). The 
Table 1 Contacts of Get5 to ribosomal proteins identified by mass spectrometry.

\begin{tabular}{|c|c|c|c|c|c|}
\hline Hit & MW (kDa) & Coverage (\%) & $p$ value & Total spectra & $\begin{array}{l}\text { His }_{6}-\text { Get5/Get5 } \\
\text { (total spectra) }\end{array}$ \\
\hline Rpl20a (eL20) & 20.4 & 62 & 0.0001 & 405 & 1.45 \\
\hline Rpl23a (uL14) & 14.5 & 43 & 0.0078 & 31 & 1.47 \\
\hline Rpl37a (eL37) & 9.9 & 25 & 0.0088 & 25 & 1.60 \\
\hline Rps19a (eS19) & 15.9 & 34 & 0.00011 & 21 & 1.47 \\
\hline Rps29a/b (uS14) & 6.7 & 70 & 0.0044 & 71 & 1.79 \\
\hline Sgt2 & 37.2 & 14 & 0.0015 & 9 & n.d. ( 0 in Get5 control) \\
\hline
\end{tabular}

Given are the conventional yeast names of ribosomal proteins and in brackets their names according to the unified nomenclature 77 . The $p$ value was determined by a one-way ANOVA test (performed in Scaffold and followed by a Benjamini-Hochberg correction) and core ribosomal proteins with a $p$ value $<0.01$ were selected according to their enrichment in the His-tagged sample with a 1.4 -fold enrichment used as a threshold (see Supplementary Dataset 1).

combined data identify two major ribosomal contacts of Get5 as proteins Rpl26 and Rpl35. Of note, Rpl26 and Rpl35 localize adjacent to each other in the proximity of the ribosomal tunnel exit (see also Fig. 3 and Supplementary Fig. 3). The reason why the crosslink of $\mathrm{His}_{6} \mathrm{Get} 5$ to Rpl35 was not identified via the mass spectrometry approach remains unclear; we note that small proteins containing a large number of positively charged lysine and arginine residues are frequently difficult to identify via mass spectrometry analysis ${ }^{31}$ (Supplementary Fig. 2d).

Get4 contacts helix $46 / 47$ of the $25 S$ ribosomal RNA. To map the binding site of Get $4 / 5$ on the ribosome in more detail, we utilized the method of crosslinking and analysis of cDNA $(\mathrm{CRAC})^{32,33}$. In these experiments, proteins expressed with a $\mathrm{His}_{6}$-TEV-ProtA-tag (HTP-tag) are crosslinked to their associated RNAs in vivo and subsequently RNA fragments protected by the HTP-tagged protein are identified (Fig. 3a). Yeast strains expressing C-terminally HTP-tagged Get4, and as controls Get3 and Sgt2, from their genomic loci were generated (Supplementary Fig. 3a, b). For reasons unknown and despite repeated attempts, the strain expressing Get5-HTP from the GET5 genomic locus displayed growth defects and a low Get5-HTP expression level (Supplementary Fig. 3a, b). Thus, plasmid encoded N- or Cterminally HTP-tagged Get 5 constructs were expressed in a $\Delta$ get5 strain (Supplementary Fig. 3c, d). Crosslinking of the HTP-tagged proteins to cellular RNAs revealed that only Get4-HTP was specifically crosslinked to RNA (Fig. $3 \mathrm{~b}$ and Supplementary Fig. 3e). The membrane area containing RNAs specifically crosslinked to Get4-HTP, and the equivalent area of the wild type lane, were excised (Fig. 3b). RNAs were eluted, reverse transcribed and cDNA libraries amplified by PCR (Supplementary Fig. 3f) were subjected to next-generation sequencing. Analysis of the relative distributions of normalized, mapped sequencing reads (Supplementary Fig. 3g) derived from different classes of RNAs revealed enrichment of tRNA sequences with Get4-HTP compared with the wild type control (Supplementary Fig. 3h, upper panel and Supplementary Table 2). The relative distribution of sequencing reads derived from different tRNA isoacceptors was, however, not significantly altered between the wild type and Get4-HTP samples (Supplementary Fig. 3i). Non-specific enrichment of tRNAs with RNA-binding proteins during CRAC was previously reported and likely reflects the high abundance of tRNAs and their accessibility for crosslinking, rather than specific interactions ${ }^{34}$. We thus did not follow up on this observation. Analysis of the relative distributions of sequencing reads among other classes of RNA revealed an increased proportion of rRNA sequences associated with Get4HTP compared with the wild type (Supplementary Fig. 3h, lower panel). Therefore, the numbers of sequencing reads in the wild type and Get4-HTP data sets mapping to each nucleotide of the $R D N 37$, encoding the initial pre-rRNA transcript containing the $25 \mathrm{~S}, 18 \mathrm{~S}$, and 5.8S rRNA sequences, were analyzed. This revealed that Get4-HTP, but not the wild type control, specifically crosslinked to a stretch of $\sim 70$ nucleotides encompassing helices h46 and h47 of 25 S rRNA domain III (Fig. 3c, d). Thus, Get4 did not form significant crosslinks with ribosomal proteins, (Supplementary Fig. 2b), however, was in close proximity to $25 \mathrm{~S}$ rRNA h46/h47 (Fig. 3d). These data suggest that the minor fraction of Get4, which was bound to ribosomes in the absence of Get5 (Fig. 1a, b) results from low-affinity binding of Get4 to rRNA.

In summary, five $60 \mathrm{~S}$ ribosomal proteins were identified as crosslinking partners of Get5 (Table 1, Fig. 3e and Supplementary Fig. 3j). Three of these, Rpl26, Rpl37, and Rpl35, form a cluster, ideally positioned to provide an extended binding surface for the Get $4 / 5$ complex in close proximity of the ribosomal tunnel exit (Fig. 3f and Supplementary Fig. 3k, l). Of particular note is the Get4/5 contact to Rpl35, as eukaryotic SRP4,35,36, nascent polypeptide associated complex (NAC) ${ }^{37,38}$, the amino peptidase $\mathrm{Map}^{39}$, and the Sec61 translocon ${ }^{40,41}$ all interact with Rpl35. Get 4 contacts h46/47 of the $25 \mathrm{~S}$ rRNA adjacent to Rpl26, which is located at the surface of the ribosome and partially within the ribosomal exit tunnel (Supplementary Fig. 3k). Additional contacts of Get5 (Rpl20, Rpl23, Rps19, and Rps29) faced the opposite side of the ribosome (Supplementary Fig. 3j) and the relevance of these contacts requires further investigation.

Get4/5 and SRP possess overlapping ribosome-binding sites. The findings described above (Figs. 2, 3e, f, Supplementary Fig. $3 \mathrm{k}, \mathrm{l})$ suggested that the ER-targeting factors Get $4 / 5$ and $\mathrm{SRP}^{4,35,36}$ have overlapping ribosome-binding sites. To test this possibility, we employed a yeast in vitro translation system, in which we generated RNCs carrying the yeast TA protein Sec22 as a nascent chain (Supplementary Fig. 4a). RNCs and ribosomeassociated factors can be affinity purified via a FLAG-tag fused to the N-terminus of the nascent chain (Supplementary Fig. 4b). In order to test the effect of the emerging TA sequence, a linker of increasing length was added to Sec22 following the C-terminal TA sequence (RNCs-Sec22+10-60), Fig. 4a and Supplementary Fig. 4a). In a first series of experiments, RNCs carrying Sec22, $\operatorname{Sec} 22+10$, or $\operatorname{Sec} 22+20$, with the TA sequence inside of the ribosomal tunnel, were tested for the binding of Get4/5 and SRP (Supplementary Fig. 4c, d). After in vitro translation, $2-3 \%$ of ribosomes were isolated as RNCs carrying FLAG-tagged nascent chains (Supplementary Fig. $4 c$ and ${ }^{42}$ ). When RNCs carried the most N-terminal 100 residues of the soluble protein Pgk1 as a nascent chain $\sim 2 \%$ of Get $4 / 5$ and $0.3 \%$ of SRP were associated 
a

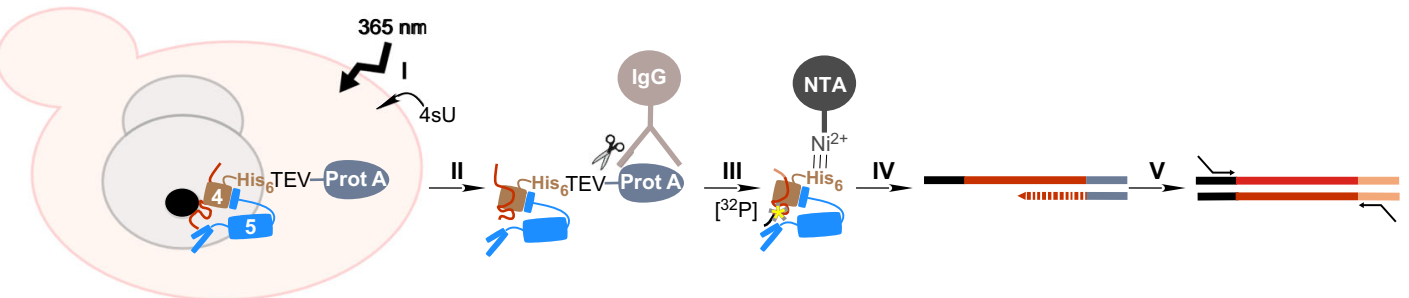

b

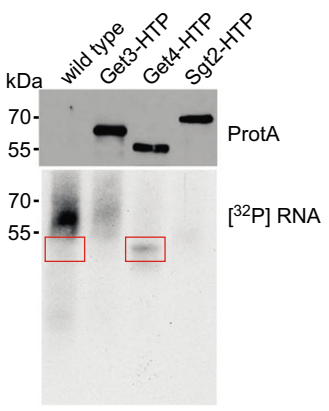

C

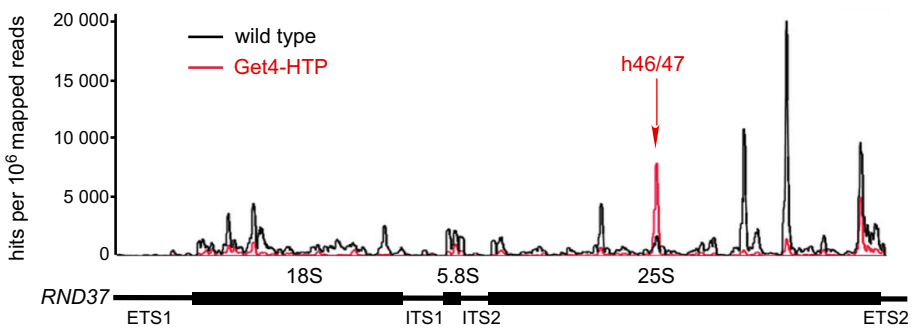

d

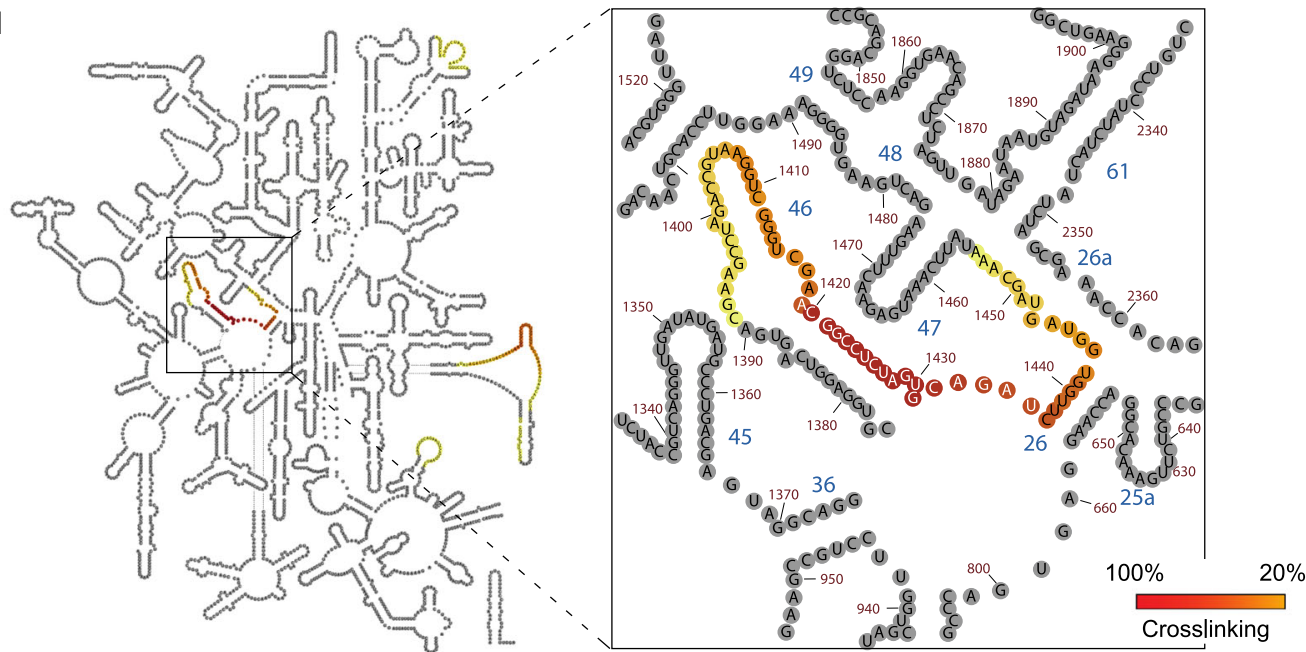

e
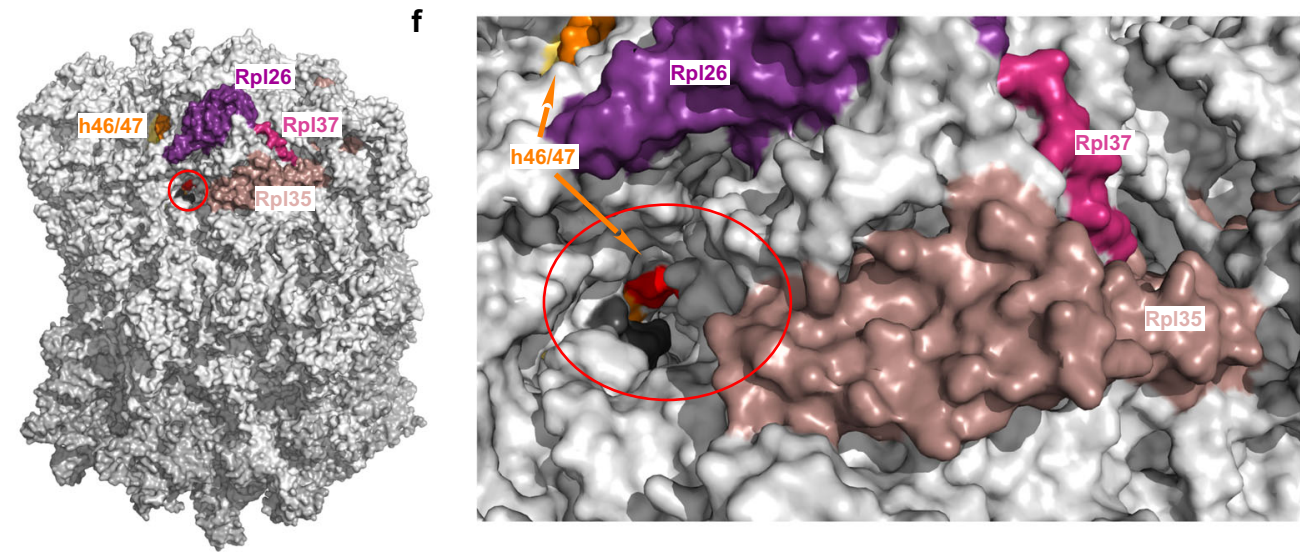

(Supplementary Fig. 4c). When RNCs, however, carried nascent Sec22 with the TA sequence inside of the tunnel, binding of Get4/ 5 was increased to $3-6 \%$ and that of SRP to 2-3\% (Supplementary Fig. 4c). Binding of Get $4 / 5$ to RNCs-Sec 22 or RNCs-Sec $22+60$ was independent of Sgt2 or Get3, confirming that Get4/5 was bound to RNCs carrying FLAG-tagged nascent chains (Supplementary Note 2 and Supplementary Fig. 4e, f). The combined data confirm previous findings indicating that a TA sequence/TM domain inside of the ribosomal tunnel enhances the affinity of Get4/5 and SRP for ribosomes ${ }^{4,18,24}$. Moreover, the side-by-side analysis of Get4/5 and SRP (Supplementary Fig. 4c) suggested that the two targeting factors display similar affinities for ribosomes containing a TA sequence within the ribosomal tunnel. To further substantiate competition between Get4/5 and SRP, we 
Fig. 3 Ribosome-bound Get4 contacts h46/47 of the 25S rRNA. a Crosslinking and analysis of cDNA (CRAC). (I) Yeast expressing His 6 -TEV-ProtAtagged (HTP-tagged) Get4 (Get4-HTP) was grown in the presence of 4-thiouracil (4sU) and was then crosslinked via irradiation at $365 \mathrm{~nm}$. (II) Get4-HTPcontaining complexes were purified under native conditions, RNA was trimmed, and TEV protease-mediated cleavage was performed. (III) RNA fragments were $\left[{ }^{32} \mathrm{P}\right]$-labeled, adaptors were ligated, $\mathrm{His}_{6} \mathrm{Get} 4$-containing crosslink products were denatured, and purified via Ni-NTA. (IV) cDNA libraries were generated, (V) amplified by PCR, and sequenced. Yeast cell (pink), ribosome (gray), polypeptide tunnel exit (black), crosslinked rRNA (red), Get4/5 as in Fig. 2a. b Get4 contacts RNA. ProtA-purified, trimmed, [32P]-labeled, and crosslinked material (ProtA immunoblot, upper panel) and $6 \mathrm{~h}$ autoradiography $\left(\left[{ }^{32} \mathrm{P}\right]\right.$-labeled RNA, lower panel) of the indicated strains. Membrane regions, which were employed for further analysis as shown in panels $\mathbf{c}$ and $\mathbf{d}$ are boxed in red. c Get4 contacts $25 \mathrm{~S}$ rRNA h46/h47. Sequencing reads mapping to nucleotides of the S. cerevisiae RDN37 rDNA sequence. Get4-HTP (red) and wild type (black). Shown is a representative experiment of two biological replicates (accession code GSE151664). PCR amplification (panel a, step V) was 35 cycles for wild type, and 24 cycles for Get4-HTP. d Heat map of Get4 contacts to 25S rRNA. 25S rRNA secondary structure and zoom into the rRNA region crosslinked to Get4 ${ }^{72}$. Red $(100 \%)$ indicates the maximum number of reads obtained, lower numbers of reads ( $\left.>20 \%\right)$ are shown in shades of orange-yellow. e Contacts between Get4/5, ribosomal proteins, and rRNA. Surface representation of the yeast 80S ribosome (PDB 4V8878). 60S (light gray) and 40S (gray). Contacts of Get5 to ribosomal proteins close to the tunnel exit: Rpl26 (deep purple), Rpl35 (dirty violet), Rpl37 (hot pink). A red circle indicates the ribosomal tunnel exit. Additional contacts of Get5 are shown in Supplementary Fig. 3j and Table 1. f Zoom into the ribosomal tunnel. Color code as in panel e. The tunnel exposed $\beta$-sheet of Rpl17 is indicated in black. Contacts of Get4 to h46/h47 of the $25 \mathrm{~S}$ rRNA are as in $\mathbf{d}$ (see Supplementary Fig. 3k).

employed the SRP substrate Dap2, a type 2 membrane protein, the early targeting steps of which were characterized in detail ${ }^{18,42,43}$. SRP binds with low affinity to RNCs carrying the Nterminal 60 residues of Dap2 (RNCs-Dap2-60) ${ }^{18}$, which contain the Dap2 TM domain (residues 30-45) inside of the ribosomal tunnel (Supplementary Fig. 4g). To test whether Get $4 / 5$ affected this low-affinity binding of SRP to RNCs-Dap2-60, SRP binding was studied in the presence or absence of Get4/5 (Fig. 4b). The occupancy of RNCs-Dap2-60 with SRP was significantly increased when Get4/5 was absent from the reaction (Fig. 4b). We conclude that Get4/5 and SRP compete for ribosome binding when a TM domain is inside of the ribosomal tunnel (Supplementary Fig. 4c, Fig. 4b, and Supplementary Note 3).

The analysis was then extended to RNCs bound to Sec22 with longer C-terminal extensions (Fig. 4a). Exposure of the TA sequence outside of the ribosomal tunnel resulted in reduced binding of Get4/5, while at the same time binding of SRP was strongly increased (Fig. 4c). This observation suggests that Get4/5 cannot bind if SRP is bound to RNCs exposing a TM domain. To test this directly, RNCs were generated in an in vitro translation reaction lacking SRP (Fig. 4d and Supplementary Fig. 4f). When SRP was absent, the amount of Get $4 / 5$ bound to RNCs remained high, even when the TA sequence was accessible (Fig. 4d). We conclude that tight binding of SRP to ribosomes exposing an SRP recognition sequence disfavors ribosome-binding of Get $4 / 5$.

Ribosome-bound Get4/5 recruits Sgt2 to facilitate TA protein transfer to Sgt2. The Get $4 / 5$ complex forms an elongated heterotetramer ${ }^{11,28}$, which interacts with Sgt 2 via the ubiquitinlike (UBL) domain of Get5 $5^{11,44}$. As Sgt2 is the GET pathway component, which first captures newly synthesized TA proteins, we asked whether ribosome-bound Get $4 / 5$ is able to interact with Sgt 2 and by that, positions Sgt 2 in close proximity of the tunnel exit (Supplementary Fig. 4h). FLAG-tag pull-down experiments with RNCs-Sec22 (TA sequence within the ribosomal tunnel) revealed that the amount of ribosome-associated Sgt 2 was close to the detection limit of the Sgt 2 antibody, and the result remained ambiguous (Fig. 4e, lane 1 and 2). We reasoned that if Get4/5 indeed recruited Sgt2, a higher occupancy of RNCs with Get4/ 5 should result in enhanced recruitment. To test this possibility, purified $\mathrm{His}_{6} \mathrm{Get} 4 / \mathrm{Get} 5$ was added to pull-down reactions (Fig. 4e and Supplementary Fig. 4i, j). Indeed, when the occupancy of RNCs with Get $4 / 5$ was high, significantly more Sgt 2 was associated with RNCs-Sec22, even though the concentration of Sgt 2 in the reaction remained unaltered (Fig. 4e, lanes 3 and 4). A similar increase in Sgt2 recruitment was observed when purified $\mathrm{His}_{6}{ }^{-}$ Get4/Get5 was added to RNCs-Sec22 + 60, which expose the TA sequence outside of the ribosomal tunnel (Fig. 4e, lanes 5-8). Binding of Get4/5, as well as Get4/5-dependent binding of Sgt2, was also tested with RNCs carrying Sed5 or Bos1 (Supplementary Fig. $4 \mathrm{k}$ ). RNCs-Sed $5+60$ or - Bos $1+60$ recruited Get $4 / 5$ and a low amount of Sgt2, close to the detection limit of the Sgt2 antibody, in a wild type translation extract (Supplementary Fig. $4 \mathrm{k}$ ). When $\mathrm{His}_{6}$ Get $4 /$ Get5 was added to the reaction, binding of Sgt 2 was strongly enhanced (Supplementary Fig. 4k). Consistent with enhanced association of Get $4 / 5$ with RNCs-Sec $22+$ 60 in the absence of SRP (Fig. 4d, f), Sgt2 recruitment to RNCsSec $22+60$ was increased in the absence of SRP, and was further stimulated about 3-fold when purified $\mathrm{His}_{6}$ Get4/Get5 was added to the reaction (Fig. $4 \mathrm{f}, \mathrm{g}$ ).

Proximity of nascent Sec22 (with TA inside of the ribosomal tunnel) and Sec $22+60$ (with TA outside of the ribosomal tunnel) to SRP, Get4/5, and Sgt2 was further analyzed by crosslinking (Supplementary Fig. 5a). SRP did not contact nascent Sec22, however, was in direct contact with nascent Sec22 +60 (Fig. 5a, see Fig. 4c for occupancy of RNCs-Sec22/Sec22 + 60 with SRP). Thus, a bona fide TA sequence served as an SRP recognition sequence when it was exposed as a nascent chain outside of the ribosomal tunnel. We did not detect crosslinks between Get4 or Get5 and nascent Sec22 + 60 (Supplementary Fig. 5b, c), or between Sgt 2 and nascent Sec22, even when the occupancy of RNCs-Sec22 with Get4/5 and Sgt2 was high (Supplementary Fig. 5d, see Fig. 4e for occupancy of RNCs-Sec22 with Sgt2). However, when ribosomes carried nascent $\operatorname{Sec} 22+60$, and thus the TA sequence was exposed, Sgt 2 was crosslinked to the nascent chain (Fig. 5b, c). In a wild type translation extract, crosslinking between Sgt 2 and nascent $\operatorname{Sec} 22+60$ was weak, in some experiments barely above the detection limit (Fig. 5b, c). Crosslinking between Sgt 2 and nascent Sec $22+60$ was significantly increased when SRP was absent (Fig. 5b) or when the occupancy of RNCs-Sec $22+60$ with Get4/5 was high (Fig. 5c). Thus, the extent of crosslinking between Sgt2 and nascent Sec22 +60 correlated with the occupancy of RNCs-Sec $22+60$ with Get4/5/Sgt2 (Fig. 4e, f). The ribosome binding and crosslinking data shown in Figs. 4 and 5 are consistent with the wellcharacterized properties of SRP, which efficiently interacts with nascent TM domains, and Get $4 / 5$, which does not interact with TA sequences directly $1,5,15,16$. In addition, the data reveal that Sgt 2 was recruited to RNCs via Get $4 / 5$ even before the TA sequence emerged from the tunnel (Fig. 4e), but moved into close proximity to the nascent TA protein only after the TA sequence had emerged from the ribosomal tunnel (Fig. 5b, c).

In vivo, TA sequences do not exit the ribosomal tunnel during ongoing translation, and emerge only after translation termination 
a

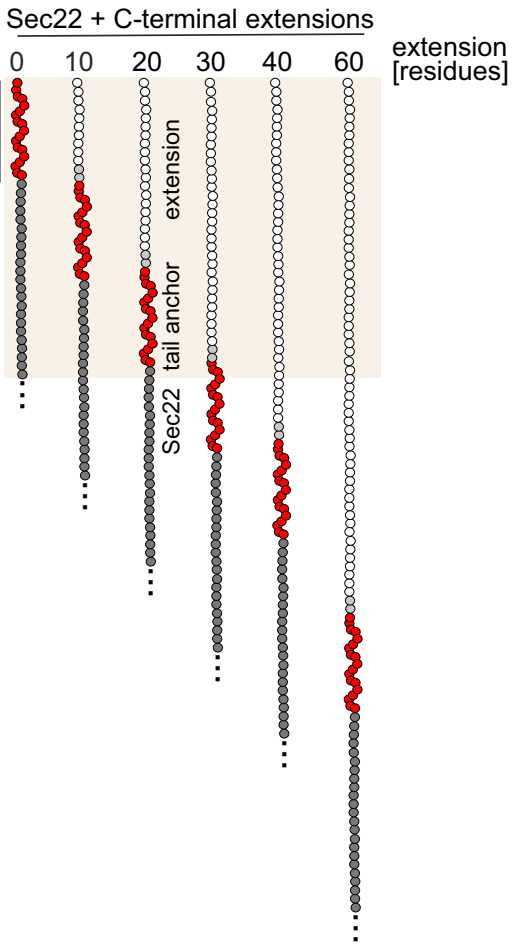

b

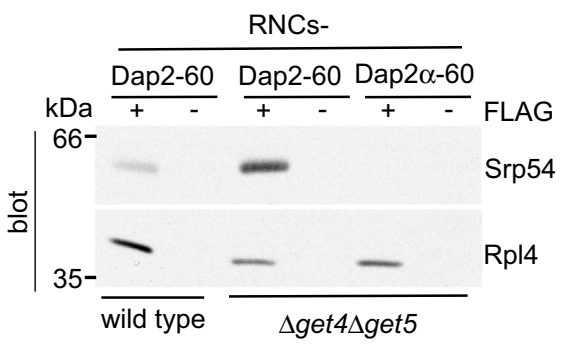

f

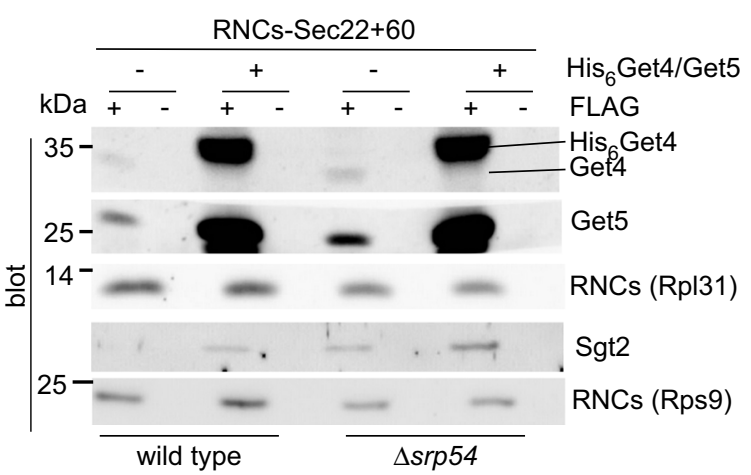

(Supplementary Fig. 5e). To investigate TA recognition during peptide release, we produced a ribosome-released TA protein by programming the yeast translation extract with an mRNA encoding full-length Sec22 including the stop codon (Supplementary Fig. 5f). We first assessed how much Sgt2 or SRP was bound to released Sec22 compared with nascent Sec22 +60 . To control for equal loading, released Sec22 and nascent Sec22 +60 were labeled with $\left.{ }^{35} \mathrm{~S}\right]$-methionine (Fig. $5 \mathrm{~d}$ ). Consistent with previous data $1,5,15,16$, Sec22 released upon translation was mainly bound to Sgt2 (Fig. 5d and Supplementary Fig. 5g and h), whereas nascent Sec22 + 60 was mainly associated with SRP (Fig. 5d). These data
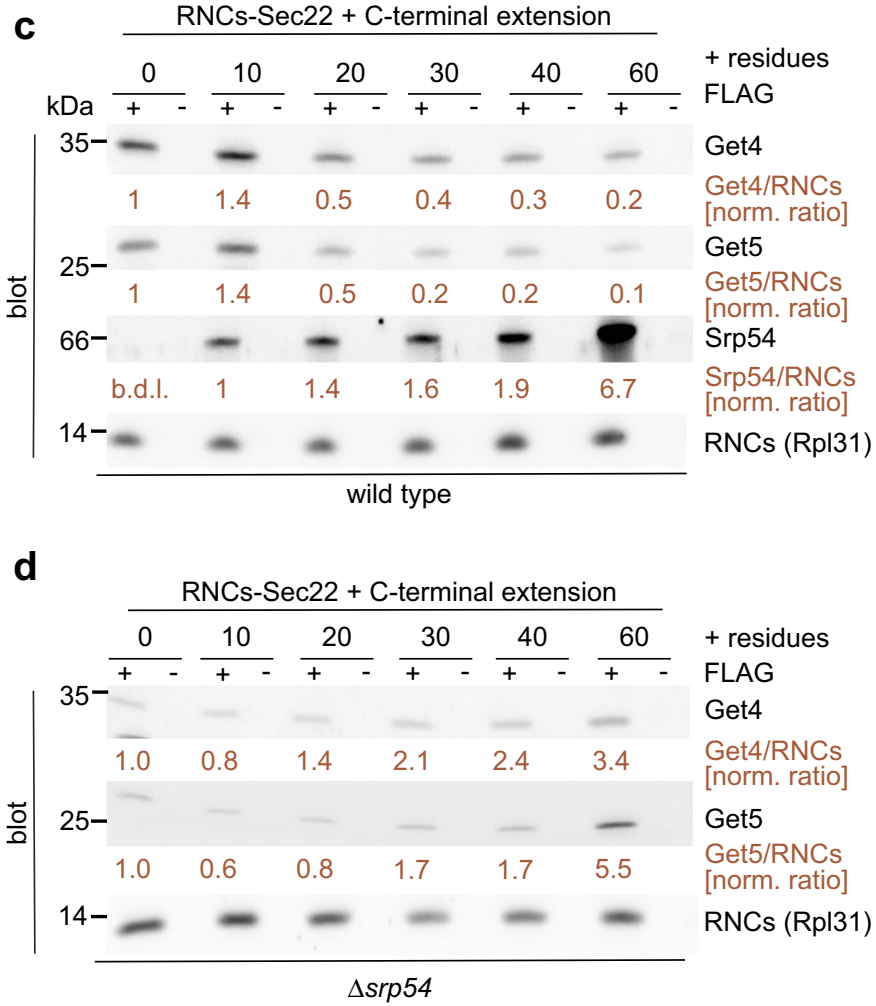

e

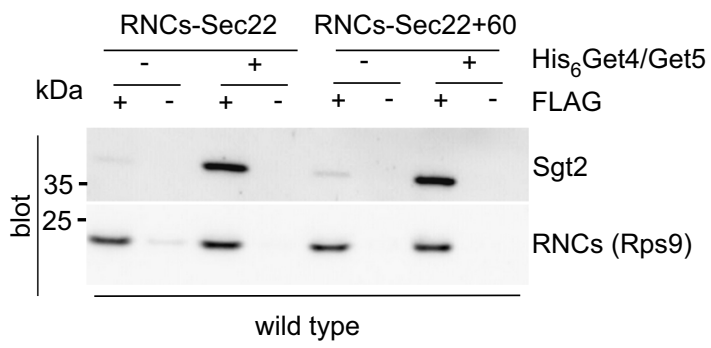

g

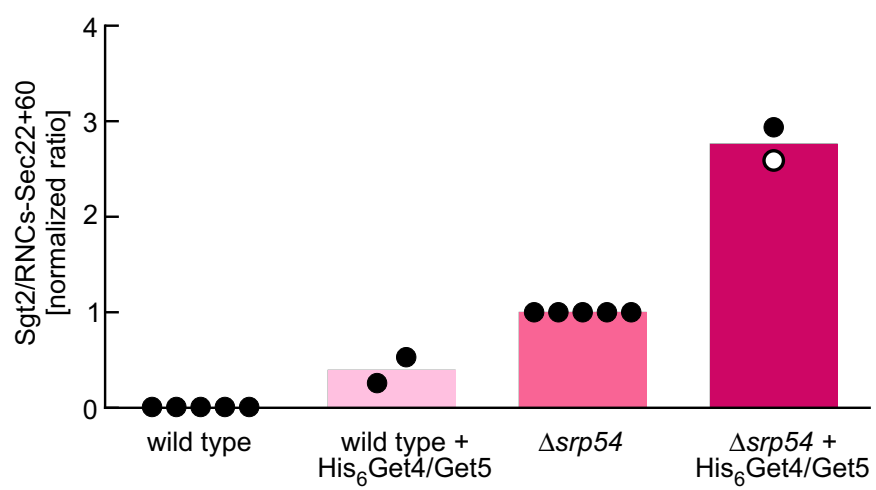

raised the question of whether the Get4/5-dependent recruitment of Sgt2 to ribosomes (Fig. 4) facilitated the capture of released Sec 22 by Sgt 2 . As Get $4 / 5$ has an additional role during a later step of the GET pathway when the TA protein is transferred from Sgt2 to Get3, the analysis was performed in the absence of Get $3{ }^{45}$. To that end, capture of Sec22 by Sgt2 was analyzed in a $\Delta$ get 3 translation extract with or without addition of purified $\mathrm{His}_{6} \mathrm{Get} 4 /$ 5 , and in a $\Delta$ get $3 \Delta$ get $4 \Delta$ get5 translation extract (Fig. 5e and Supplementary Fig. 4f). In this setup, addition of purified $\mathrm{His}_{6}$ Get $4 /$ Get 5 consistently enhanced binding of Sec22 to Sgt2 by more than twofold (Fig. 5e, f). Moreover, a smaller fraction of 
Fig. 4 Competition between Get4/5 and SRP with respect to ribosome-binding. a Schematic representation of in vitro analyzed RNCs. The C-terminus of the tail-anchor (TA) protein Sec22 was extended by 10-60 residues. Estimate of the nascent chain covered by the ribosomal tunnel ${ }^{43}$ (beige), Sec22 (dark gray, tail-anchor in red, C-terminal extension in light gray and white; for details see Supplementary Fig. 4a). b SRP binding to RNCs-Dap2-60 is enhanced in the absence of Get4/5. FLAG-tag pull-down reactions (see Methods and Supplementary Fig. 4b) with FLAG-tagged (+FLAG) or non-tagged ( - FLAG) Dap2-60 or Dap2 $\alpha-60$ (control without TM domain ${ }^{18}$ ) were generated in translation extracts as indicated (Supplementary Fig. 4f). The relative amount of RNCs (Rpl4) and bound SRP (Srp54) was assessed by immunoblotting. c-d SRP reduces binding of Get4/5 to RNCs when the TA sequence is exposed outside of the ribosomal tunnel. RNCs carrying FLAG-tagged Sec22 variants (a) were generated in a wild type (c) or $\Delta$ srp54 translation extract (d). FLAGpull-down reactions were performed as in $\mathbf{b}$ and intensities of immunostained bands were determined densitometrically. Relative ratios of Get4/Rpl31, Get5/Rpl31, and Srp54/Rpl31 were calculated and the ratio obtained for RNCs-Sec22 was set to 1; as the intensity of Srp54 on RNCs-Sec22 was below the detection limit (b.d.I.), RNCs-Sec22+10 were used for Srp54 normalization. See also Supplementary Fig. 4c. e Recruitment of Sgt2 to RNCs-Sec22 depends on Get4/5. FLAG-tag pull-down reactions were performed as in $\mathbf{b}$. As indicated (+), purified His 6 Get4/Get5 ( $2 \mu \mathrm{M})$ was added prior to the translation reaction. f Sgt2 recruitment to RNCs-Sec22+60 is enhanced at high ribosome occupancy with Get4/5, or when SRP is absent. FLAG-tag pull-down reactions were performed and analyzed as in $\mathbf{b}$ with $\mathrm{His}_{6} \mathrm{Get} 4 / \mathrm{Get} 5$ addition as in e. RNCs (Rps9 or Rpl31). $\mathbf{g}$ Quantification of Sgt2 recruitment to RNCsSec22+60. Ribosome-binding of Sgt2 was analyzed as in $\mathbf{f}$. Shown are the normalized mean of five (wild type and $\Delta$ srp54) and two (wild type and $\Delta$ srp54 $+\mathrm{His}_{6} \mathrm{Get} 4 / \mathrm{Get5}$ ) biological replicates (bars) and the result of each experiment (dots) (see Methods).

Sec22 was bound to Sgt 2 when Get4/5 was absent from the reaction (Fig. 5e, f). We conclude that Get $4 / 5$ is not only required for the transfer of TA proteins from Sgt2 to Get3 $3^{1,5,10,15,16,45}$, but prior to that, Get $4 / 5$ facilitates efficient transfer of TA proteins to Sgt2 upon ribosome-release.

In vivo binding of Sgt 2 to ribosomes depends on Get4/5. The above findings suggested that in living cells, a fraction of Sgt2 could be ribosome-bound. Previous analysis failed, however, to provide evidence for a ribosome-bound pool of Sgt $2{ }^{24}$. Based on the above data, we revisited the question of ribosome-binding of Sgt2 in vivo. For that purpose, we increased sample loading and further optimized immunoblotting with the highly specific, but poorly sensitive Sgt2 antibody (Fig. 6a). A minor portion of Sgt2 was detected in ribosomal fractions of a wild type cell extract under low salt conditions and was released by high salt treatment (Fig. 6a). Moreover, when Get4/5 was overexpressed the fraction of ribosome-bound Sgt 2 was increased (Fig. 6a, b). Ribosome profile analysis in the wild type strain, a strain overexpressing Get4/5, and a $\Delta g e t 4 \Delta$ get 5 strain was conducted to further validate Get4/5-dependent binding of Sgt2 to translating ribosomes (Fig. 6c). To enhance detection of factors bound to polysomes, a modified ribosome profile protocol, which combines the polysome fractions prior to analysis was developed (see Methods). In a total extract derived from wild type a small fraction of Sgt 2 comigrated with ribosomes (Fig. 6d, fractions 5-19), in the extract from Get4/5 overexpressing cells the fraction of Sgt 2 in ribosome fractions was increased more than twofold (Fig. 6e, fractions 5-19), and when Get4/5 was absent, the amount of Sgt 2 associated with ribosomes was strongly reduced (Fig. 6f, fractions 5-19). The fraction of SRP associated with ribosomes was largely unaffected by the level of Get4/5 (Fig. $6 \mathrm{~d}$, f, fractions 5-19); this finding is in line with the observation of Get4/5-independent binding of SRP to the ribosome. Increasing the expression level of Get $4 / 5$ resulted in a significantly higher occupancy of ribosomes with Get4/5, which in turn resulted in a higher occupancy of ribosomes with Sgt2 (Fig. 6a and d-f). Together, these data support the requirement of Get4/5 for ribosome recruitment of Sgt2.

\section{Discussion}

In this work, we show how newly synthesized tail-anchored proteins are captured upon their emergence from the ribosomal tunnel. Five major conclusions can be drawn from the results of this study: (i) Get4/5 binds directly to the ribosome via the Get5 subunit; (ii) Get4/5 and SRP have overlapping binding sites and compete with each other for binding to the ribosome; (iii) ribosome-bound Get4/5 recruits Sgt2; (iv) ribosome-associated
Sgt2 contacts exposed TA sequences; and (v) Get4/5-dependent ribosome recruitment of Sgt 2 improves capture of TA proteins released to the cytosol upon translation termination.

Get5 mediates binding of Get4/5 to the ribosome. Get5 has a modular architecture, which allows it to interact with different partner proteins. The N-terminal domain of Get5 stably binds to its partner Get4, whereas the adjacent UBL domain provides the binding site for the interaction with Sgt2, and the C-terminal domain is responsible for self-assembly into a stable homodimer ${ }^{11,46}$. Our analysis reveals that, in addition, Get5 contacts ribosomal proteins Rpl26 and Rpl35 close to the ribosomal tunnel exit. Which domain of Get5 mediates binding to the ribosome is currently unknown. We consider it unlikely that ribosome binding involves the N-terminal or the UBL domains, because ribosome-bound Get5 is in a complex with Get 4 and can interact with Sgt2. A possible candidate is the C-terminal homodimerization domain, the function of which is presently not understood ${ }^{11}$. As the C-terminal domain of Get 5 is negatively charged, it seems possible that electrostatic interactions mediate binding to positively charged patches within its ribosomal contact site (Supplementary Fig. 31). Interestingly, the homodimerization domain of Get5 is not conserved in the mammalian Get5 homolog UBL4A ${ }^{11}$. This would be consistent with the observation that mammalian UBL4A does not directly bind to ribosomes ${ }^{19}$ (see also below).

Ribosome binding of Get4/5 versus SRP: competition modulated by the emerging nascent chain. The amounts of SRP $\left(\sim 8000\right.$ molecules per cell $\left.{ }^{42,47}\right)$ and Get $4{ }_{2}$ Get $5_{2}(\sim 5000$ molecules per cell ${ }^{47}$ ) in living yeast cells are similar. Both proteins are much less abundant than cytosolic ribosomes, which are present at $\sim 300,000$ molecules per yeast cell ${ }^{42}$. This stoichiometry suggests that Get4/5 has to scan ribosomes in order to identify those carrying cognate nascent substrates, as it is well documented for SRP $4,21,48$. SRP and Get4/5 display surprisingly similar affinities for vacant ribosomes, with a $K_{\mathrm{d}}$ of $110 \pm 40 \mathrm{nM}$ for Get $4 / 5$ (this study) and $71 \pm 36 \mathrm{nM}^{49}$ or $120 \pm 29 \mathrm{nM}^{50}$ for SRP. Binding of Get4/5 and SRP to the ribosome is enhanced when a nascent TA sequence/TM domain enters the exit tunnel $(4,18,24$ and this study). Furthermore, the affinities of Get4/5 and SRP for translating ribosomes with a TA sequence/TM domain inside the exit tunnel resemble each other. These binding properties allow the two targeting factors to freely bind and dissociate from ribosomes in dynamic cycles and scan for TM domains emerging inside of the tunnel. Once a nascent TM domain becomes exposed outside of the tunnel, SRP binds to it with $>1000$-fold higher affinity ${ }^{49}$, 

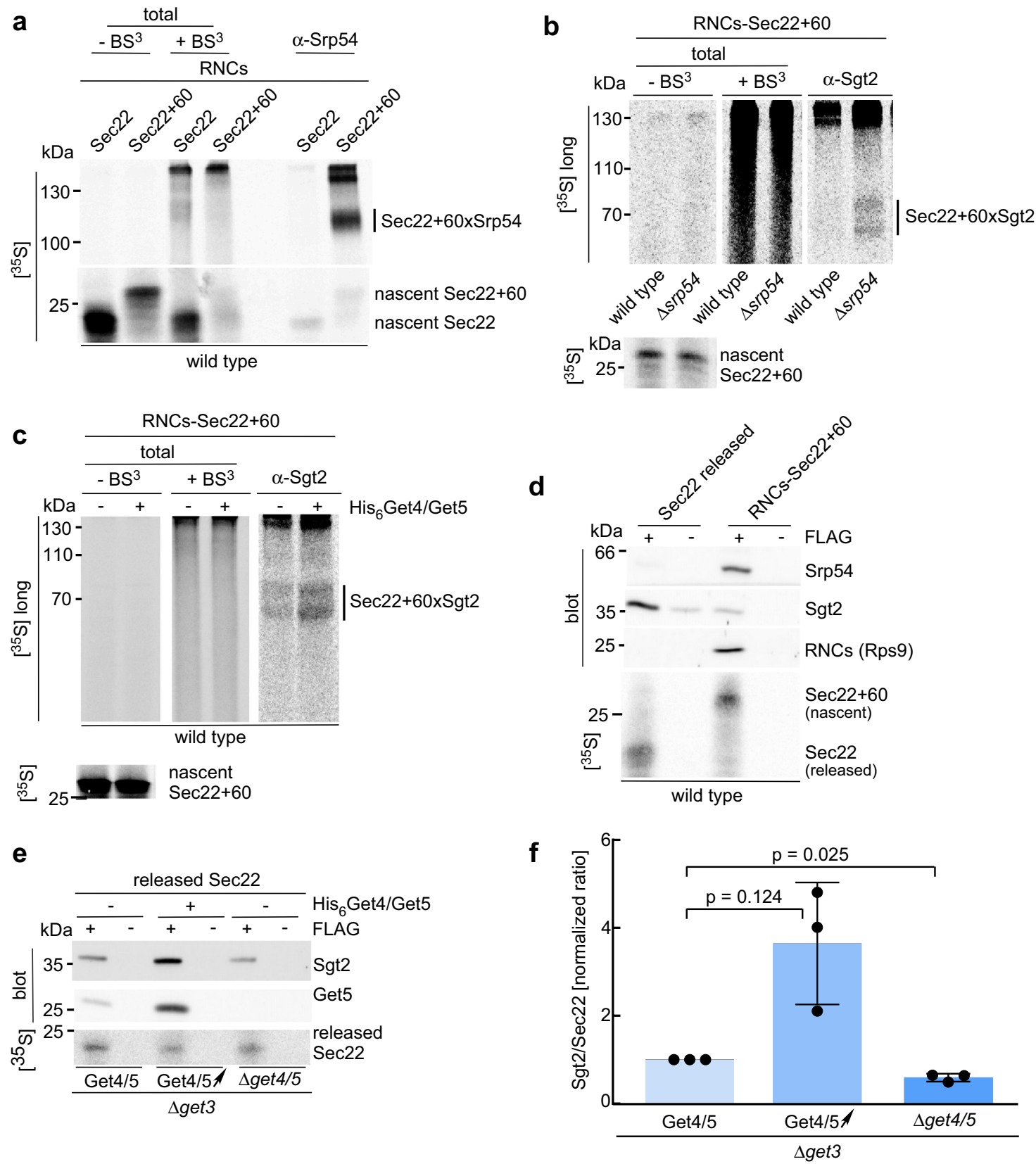

Fig. 5 Ribosome-bound Sgt2 is in contact with a nascent TA sequence. a SRP contacts the TA sequence of nascent Sec22+60 exposed outside of the ribosomal tunnel. RNCs carrying radiolabeled nascent Sec22 or Sec22+60 were crosslinked with BS $^{3}$ and SRP was affinity purified with $\alpha$-Srp54. Total $(5 \%)$ translation reactions before $\left(-\mathrm{BS}^{3}\right)$ or after $\left(+\mathrm{BS}^{3}\right)$ crosslinking. See Supplementary Fig. 5 a and Methods. Shown is an autoradiograph; Sec22 $+60 \times$ Srp54 represents the crosslink product between Srp54 and nascent Sec22+60. b Sgt2 contacts nascent Sec22+60 when SRP is absent from the reaction. Radiolabeled RNCs-Sec22+60 generated in wild type or $\Delta$ srp54 translation extracts were analyzed as detailed in a using $\alpha$-Sgt2 for affinity purification of crosslink products. Sec22+60xSgt2 represents crosslink products between Sgt2 and nascent Sec22+60. c Sgt2 contacts nascent Sec22+60 when RNCs are highly occupied with Get4/5. Radiolabeled RNCs-Sec22+60 were analyzed with or without purified His 6 Get4/Get5 ( $2 \mu M$ )

(Supplementary Fig. 4i). d Ribosome-released Sec22 is mainly bound to Sgt2, whereas nascent Sec22+60 is mainly bound to SRP. FLAG-tag pull-down reactions were performed with radiolabeled FLAG-tagged Sec22 and RNCs-Sec22+60 (Supplementary Fig. 4b and Supplementary Fig. 5f). Samples containing similar amounts of released Sec22 or RNCs-Sec22+60 were analyzed for binding of SRP (Srp54) or Sgt2 via immunoblotting. $\alpha$-Rps9 was employed to detect RNCs. e Ribosome-bound Get4/5 improves the capture of released Sec22 by Sgt2. Radiolabeled released Sec22 was generated in $\Delta$ get3 or $\Delta$ get3 $\Delta$ get $4 \Delta$ get5 translation extracts (Supplementary Fig. $4 \mathrm{f}$ ). When indicated (+), purified $\mathrm{His}_{6} \mathrm{Get}_{4} / \mathrm{Get} 5$ ( $\left.2 \mu \mathrm{M}\right)$ was added prior to the translation reaction. FLAG-tag pull-down reactions were performed and equal amounts of affinity purified, released Sec22 were analyzed for Sgt2 and Get4/5 (Get5). f Quantification of the capture of released Sec22 by Sgt2. The relative efficiency with which Sgt2 was bound to released Sec22 (e) was determined (see Methods). Shown is the mean of three independent experiments (bars), with the standard deviation, and the result of each experiment (dots). $p$ values were calculated by one-way ANOVA with GraphPad Prism. 


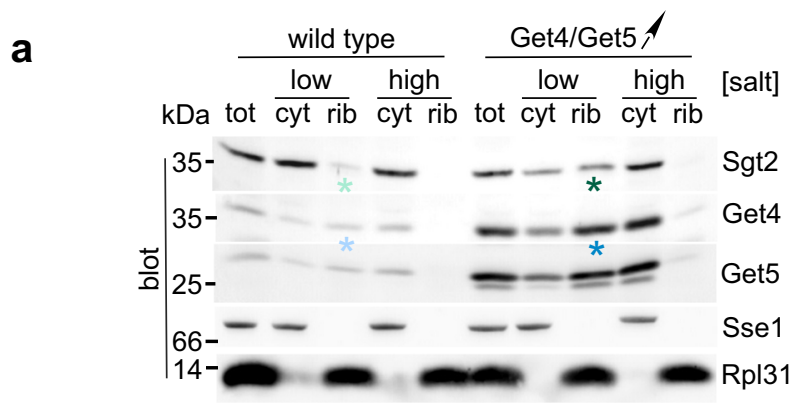

d

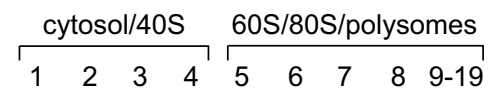
kDa tot $\overline{\text { cyt rib }} \overline{\text { cyt rib }}$ tot $\overline{\text { cyt rib }} \frac{\text { cyt rib }}{\text { cys }}$

\section{b}
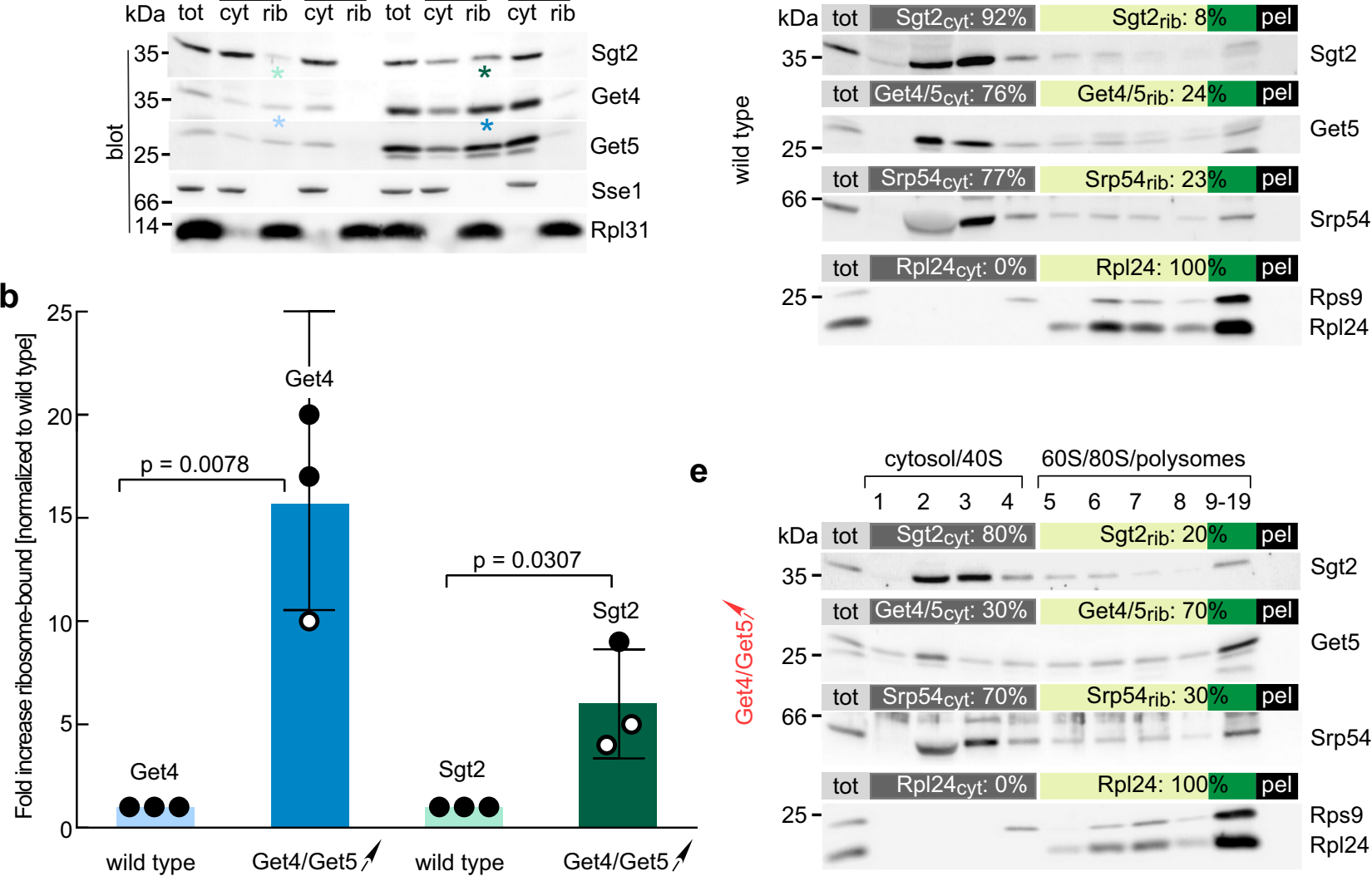

e
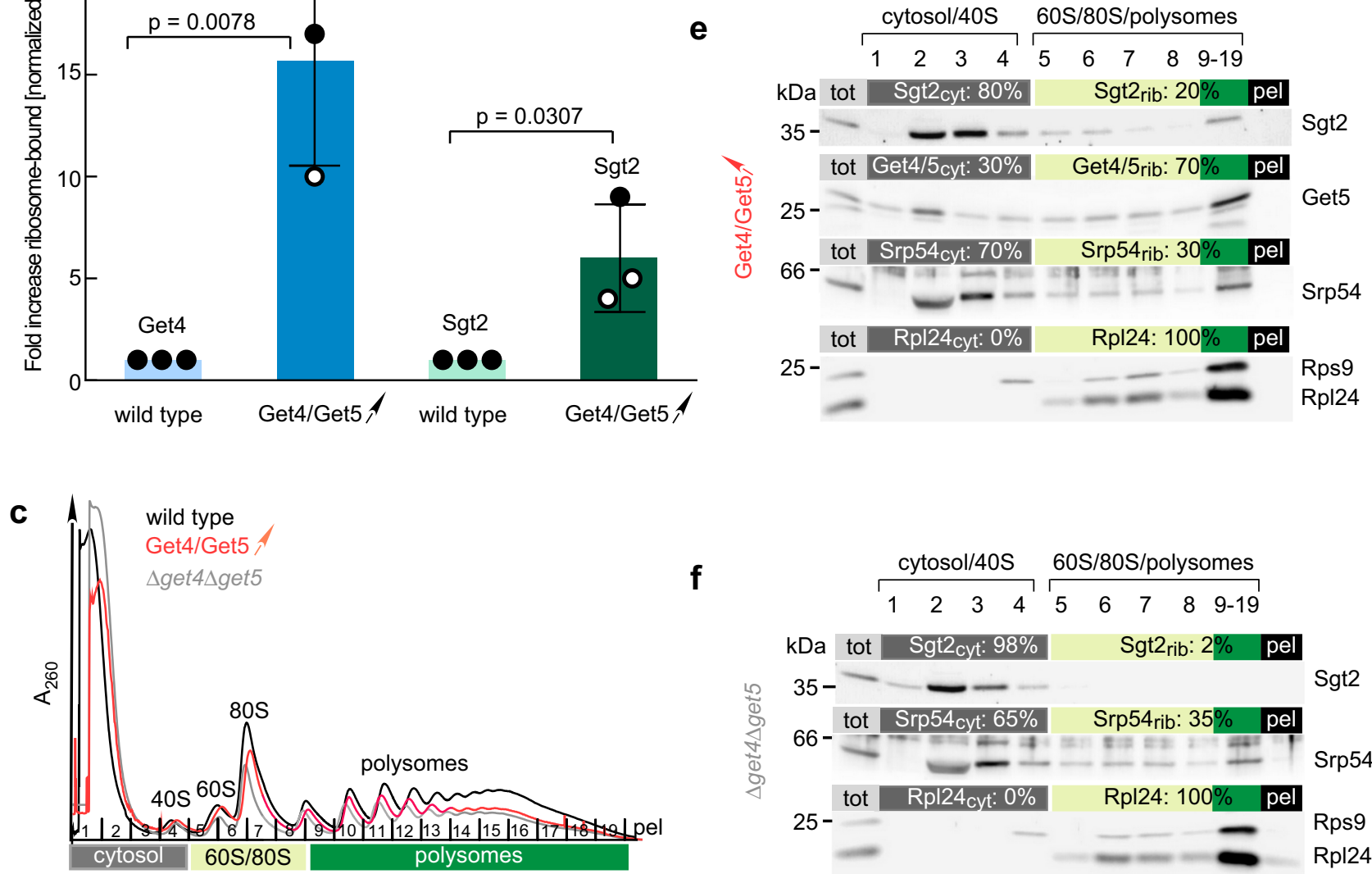

f
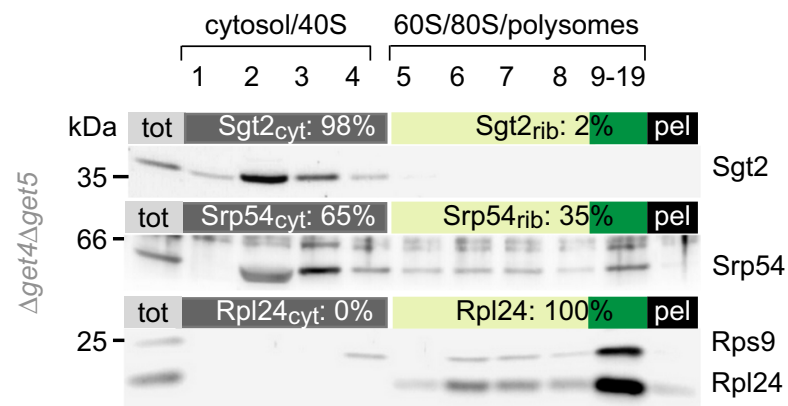

Fig. 6 Sgt2 is recruited to translating ribosomes via Get4/5 in vivo. a Sgt2 is associated with ribosomes in a Get4/5-dependent manner. Total cell extract (tot) of wild type or a strain overexpressing Get4/5 (Get4/Get5 $\uparrow$ ) was analyzed as described in Fig. 1a. Aliquots were analyzed by immunoblotting with $\alpha$-Get4, $\alpha$-Get5, $\alpha$-Sgt2, $\alpha$-Rpl31 (ribosomal marker), and $\alpha$-Sse1 (cytosolic marker). Colored asterisks indicate the bands analyzed in $\mathbf{b}$. $\mathbf{b}$ Ribosome occupancy with Sgt2 is enhanced upon overexpression of Get4/5. Ribosome-binding of Get4 and Sgt2 under low salt conditions was analyzed as in a. Shown is the mean (bars with standard deviation) of three independent experiments (dots). Values obtained for the wild type were set to 1 . Indicated $p$ values for Get4 or Sgt2 were calculated according to a two-sided Student's $t$ test using GraphPad Prism. The color code of the bars corresponds to the asterisks in a. c Ribosome profile analysis reveals a sub-population of Sgt2 in polysome fractions, which depends on the presence of Get4/5. Sucrose density gradient centrifugation was performed with total cytosolic extract prepared from wild type (black), cells overexpressing Get4/Get5 (Get4/Get5 $\uparrow$ ) (red), or $\Delta$ get $4 \Delta$ get5 cells (light gray). Indicated in dark gray are the major cytosolic fractions and 405 particles (1-4), in light green (5-8) fractions containing 605 and 805 ribosomal particles, and dark green (9-19) polysome fractions. d-f Aliquots of individual fractions 1-8, pooled fractions 9-19, and the pellet fraction at the bottom of the gradient tube (pel) (see c) were analyzed via immunoblotting with $\alpha$-Sgt2, $\alpha$-Get5, $\alpha$-Srp54, the $40 \mathrm{~S}$ subunit ( $\alpha$-Rps9), and the 60S subunit ( $\alpha$-Rpl24) (for details see Methods). The total (tot) corresponds to $5 \%$ of the cytosolic extract loaded onto the gradient. The distribution of ribosome-bound factors between cytosol and ribosomes was estimated as follows. Intensities of immunostained bands in fractions 1-19 $(100 \%=$ total intensity of fractions 1-19) was determined for each ribosome-bound factor. The percentage of each ribosome-bound factor in the cytosolic fractions 1-4 (cyt: \%) and in the ribosomal fractions 5-19 (rib: \%) was calculated relative to the total. The color code is as in c. 


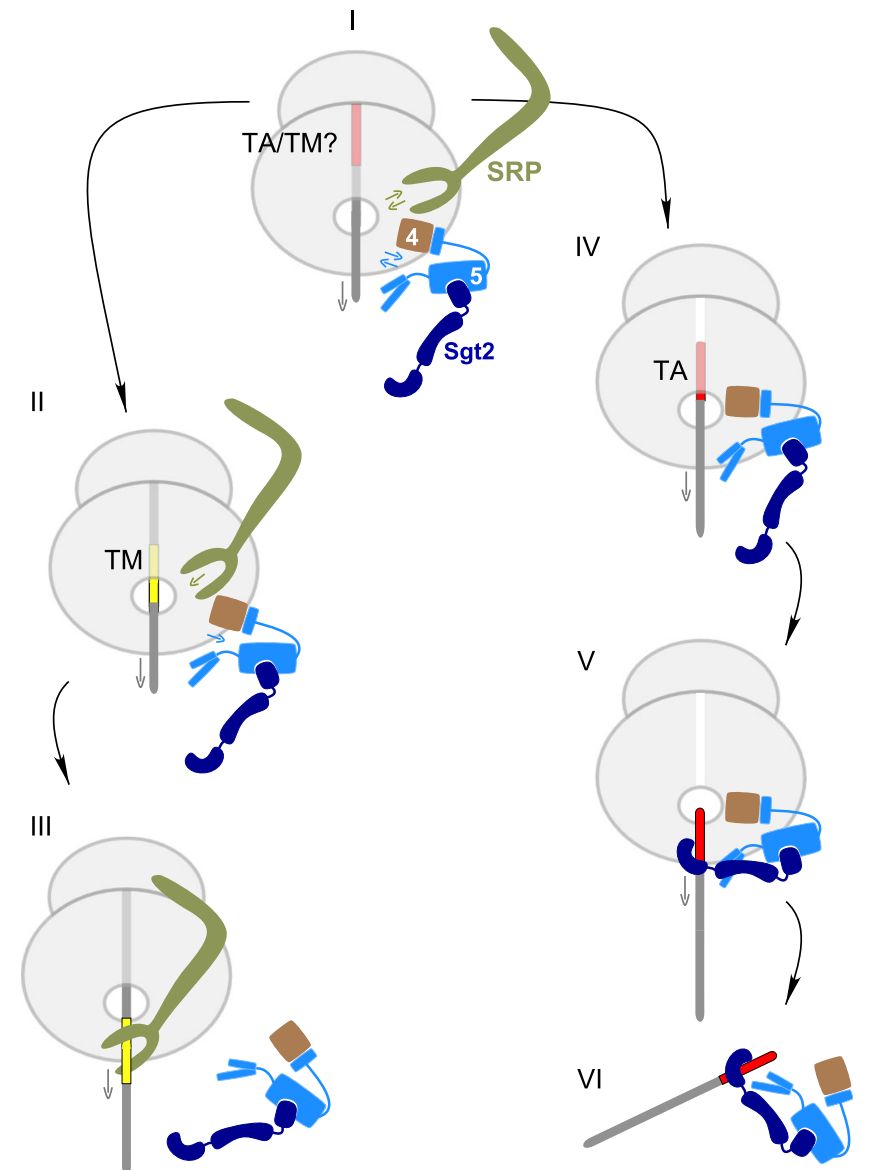

Fig. 7 Model of TM domain capture upon exit from the ribosomal tunnel. (I) A sequence with the characteristics of a hydrophobic $\alpha$-helical transmembrane domain emerges from the peptidyl transferase center and enters the ribosomal tunnel. At that point, SRP and Get4/5 cycle on and off ribosomes, as it remains unclear if the hydrophobic sequence is a TM domain (internal transmembrane domain) or a TA sequence (C-terminal transmembrane domain). (II) In case translation continues, the hydrophobic sequence remains ribosome-bound as part of the nascent chain and becomes an internal TM domain. (III) Upon exit from the tunnel, the internal TM domain is recognized by SRP. As SRP now binds with high affinity, the binding and release cycle of Get4/5, and concurrently Sgt2, is interrupted. (IV) If translation termination occurs immediately after synthesis of the hydrophobic domain, which is then termed TA sequence, SRP is not efficiently recruited to the now non-translating ribosome or the released TA sequence. (V) In this case, Get4/5, and via Get4/5 also Sgt2, continue to sample ribosomes and Sgt2 can capture the TA sequence directly upon arrival at the tunnel exit. Ribosome (light gray), nascent chain (dark gray) TA sequence (red), TM domain (yellow), Get4 (brown) Get5 (light blue), Sgt2 (dark blue), SRP (green). TA (C-terminal tail-anchor sequence) and TM (internal transmembrane domain).

whereas the affinity of Get $4 / 5$ remains largely unaffected (this study, see Supplementary Note 3).

Get4/5 functions as an adaptor of Sgt 2 at the tunnel exit. Our findings reveal that Get $4 / 5$ does not only scan ribosomes but also recruits Sgt2 into close proximity of the tunnel exit. At this earliest stage of membrane protein synthesis, both Get $4 / 5$ and SRP sample ribosomes in dynamic equilibrium (Fig. 7 stage I). Depending on the type of membrane protein, either translation continues after synthesis of the TM domain, or translation termination occurs. If translation continues, an internal TM domain emerges from the tunnel, to which SRP binds with high affinity, thereby excluding Get $4 / 5$ from scanning the ribosome (Fig. 7 stage II and III). If translation termination occurs, the TM domain turns into a TA sequence, to which SRP does not bind with high affinity, Get $4 / 5$ scanning continues (Fig. 7 stage IV), and allows Sgt2, poised at the tunnel exit, to capture the released TA sequence (Fig. 7 stage V-VI).

Transfer of TA proteins to Sgt2 after release from the ribosome. Sgt2 contains a tetratricopeptide repeat domain, which interacts with cytosolic chaperones, including the Hsp70 homolog

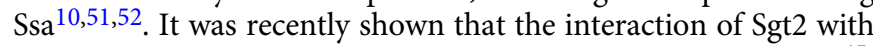
Ssa is required for the efficient capture of TA proteins by Sgt $2^{45}$. Of note, the experimental setup of that study is significantly different from ours, as it employed either purified TA proteins, or newly synthesized TA proteins generated in an Escherichia coli translation system supplemented with yeast GET components and chaperones ${ }^{45}$. As the GET pathway is not conserved in the bacterial system, we consider it unlikely that Get $4 / 5 /$ Sgt 2 binds to the E. coli ribosome. This may explain the strong tendency of the TA protein to aggregate even when Get $4 / 5$ and Sgt 2 were added to the E. coli translation reaction. Vice versa, even though Get4/ 5 significantly enhanced Sgt2-binding to a released TA protein in the yeast translation system, Get $4 / 5$ was not strictly required for the capture in our experimental system. Based on the data from the Shan $\mathrm{lab}^{45}$ and our observations, we suggest that Ssa may facilitate the Get4/5-independent, posttranslational capture of TA proteins by Sgt2 in the yeast translation system, which contains Ssa and other cytosolic chaperones in high concentrations.

The mammalian system: similarities and differences between Get4/5/Sgt2 and BAG6/SGTA. Mammalian cells express a complex consisting of GET4 (Get4 homolog), UBL4A (Get5 homolog), and BAG6 (no yeast homolog identified), which is termed the BAG6 complex ${ }^{1,11,14}$. In contrast to Get5, UBL4A does not homodimerize and does not directly bind to GET4. Instead, UBL4A and GET4 bind to BAG6 independently of each other ${ }^{1,11,14,53}$. Depletion of BAG6 results in defective TA protein capture by GET3 (Get3 homolog) ${ }^{19}$. Indeed, the BAG6 complex mediates the transfer of TA proteins from SGTA (Sgt2 homolog) to GET3 $3^{53,54}$ and thus, even though structurally different, functionally resembles the yeast Get $4 / 5$ complex ${ }^{10}$. In contrast to Get $4 / 5$, however, substrate proteins can interact directly with the BAG6 complex via its BAG6 subunit ${ }^{11,19,55,56}$. Moreover, BAG6 recruits the ubiquitin ligase RNF126, which polyubiquitinates proteins bound to BAG6 inducing subsequent degradation by the proteasome $e^{11,14,56}$. Owing to its physical interaction with GET pathway components, as well as the proteolytic system, BAG6 is involved in the triage of newly synthesized proteins between biosynthesis and degradation ${ }^{11,14}$. Although the latter function is not conserved in yeast, the BAG6 complex is recruited to ribosomes via the BAG6 subunit and in this respect resembles the yeast homolog Get $4 / 5^{19}$. The function of ribosome-bound BAG6 in mammalian cells is currently unclear. Based on the observations in this study, one could envisage that SGTA is recruited to the BAG6 complex via the UBL4A subunit. However, a recent finding reveals that this is not the case, even though SGTA binds to the mammalian ribosome ${ }^{57}$. Seemingly, in the mammalian system the interaction between SGTA and the ribosome is direct and independent of the BAG6 complex ${ }^{57}$. Similar to Sgt2, however, SGTA interacts with TM domains emerging from the ribosomal tunnel and shields them from off-pathway reactions ${ }^{57}$. Thus, although yeast and mammals employ different mechanisms to anchor the Get4/5/BAG6 complex and Sgt2/SGTA to ribosomes, the function of the ribosome-bound GET components in capturing TA proteins appears conserved. 


\section{Methods}

Yeast strains and plasmids. Yeast strains and plasmids are listed in Supplementary Table 3. BY $4741^{58}$ and $\mathrm{MH} 272-3 \mathrm{fa}^{59}$ represent the wild type strains for all mutant strains employed in this study. The FLAGRpl35 strain was previously described $^{60}$. $\Delta$ get $3 \Delta$ get $4 \Delta g e t 5$ was generated by mating of $\Delta g e t 3 a$ with $\Delta g e t 4 \Delta-$ get $5 \mathrm{a}^{24}$ followed by sporulation and tetrad dissection. The $\Delta$ get $3 \Delta$ sgt 2 strain was generated by introducing a sgt2::URA3 deletion cassette ${ }^{24}$ into the $\triangle g e t 3$ strain. Yeast genomic DNA from MH272-3fa or BY4741 was used for PCR amplification of SGT2, GET4, and GET5, for the construction of E. coli and yeast expression vectors. Yeast shuttle vectors for the overexpression of Get4, Get5, N-terminally $\mathrm{His}_{6}$-tagged $\mathrm{His}_{6} \mathrm{Get} 4$, and $\mathrm{His}_{6}$-tagged $\mathrm{His}_{6}$ Get5 are based on the pYEP and pRS series $^{61,62}$. pYEplac181-His ${ }_{6}$-Get5 was constructed by replacing the Get5 orf in pYEplac181-Get5 with $\mathrm{His}_{6} \mathrm{Get5}$, which was generated by PCR using synthetic DNA (Bio Cat) as a template (Supplementary Table 4). For in vitro translation experiments Sec $22+60$ was introduced into pSP65 (Addgene) using the EcoRI/ XbaI sites. Sec22 +60 consists of full-length Sec22, which was C-terminally fused to residues 2-61 of yeast Pgk1 via XbaI/HindIII, which introduces a two-residue linker (SR) to the C-terminus of Sec22 (Supplementary Fig. 4a). In vitro translation vectors encoding for FLAG-Sed5 $+60 /$ Sed5 +60 and FLAG-Bos $1+60 /$ Bos $1+60$ were constructed in pSP65. FLAG-Sed5 $+60 /$ Sed $5+60$ were constructed by replacing Sec22 in pSP65-Sec22 + 60/pSP65-FLAG-with Sed5 using the EcoRI/ XbaI sites. Sed $5+60$ consists of full-length Sed5, a two-residue linker (SR), and residues $2-61$ of yeast Pgk1 (Source Data file). FLAG-Bos1 +60/Bos1 + 60 were generated by PCR from a synthetic DNA template (Eurofins Genomics) (Supplementary Table 4) encoding for FLAG-Bos $1+60$. The PCR products were cloned into the SacI/HindIII site of pSP65. Bos $1+60$ consists of full-length Bos1, a tworesidue linker (LQ), and residues 2-61 of yeast Pgk1 (Source Data file). Plasmids for the in vitro translation of Dap2-60 and Dap2a-60 (Source Data file) were previously described ${ }^{18}$. For the expression of $\mathrm{His}_{6} \mathrm{Get} 4 / \mathrm{Get} 5$ in E. coli the GET4coding sequence was introduced into the NdeI/EcoRI sites of pET28a (pET28a$\mathrm{His}_{6} \mathrm{Get}_{4}$ ) and the GET5-coding sequence was introduced into the NsiI/PacI sites of pETcoco2 (pETcoco2-Get5). For the expression Get $4 / \mathrm{His}_{6} \mathrm{Get} 5$, the GET4 coding sequence was inserted into the BglII/NdeI sites and the GET5 coding sequence was inserted into the SalI/NotI sites of the pETDuet vector (pETDuetHis $_{6}$ Get5-Get4). Yeast strains expressing Get3-HTP, Get4-HTP, Sgt2-HTP, or Get5-HTP from their genomic loci were generated by homologous recombination of an HTP cassette ${ }^{33}$. For the plasmid-based expression of HTP-Get5 or Get5-HTP, the GET5-coding sequence was introduced into pRS415-based plasmids for expression of N- or C-terminally HTP-tagged proteins via the BamHI/HindIII and $\mathrm{XbaI} /$ BamHI sites, respectively ${ }^{63}$. The Get5-HTP plasmid contains a (Gly) ${ }_{4}$-Ser linker between the GET5-coding sequence and the HTP-tag. Plasmids were introduced into a $\Delta g e t 5$ background and a wild type BY4741 strain carrying the empty pRS415 plasmid (wild type e.p.) was prepared as a control (Supplementary Table 3). Primers employed in the course of the study are listed in Supplementary Table 4.

\section{Heterologous expression and purification of GET pathway components from} E. coli. pET28a-His ${ }_{6}$-Get4 and pETcoco2-Get5 were transformed into E. coli BL21 (DE3) and expression was induced at $25^{\circ} \mathrm{C}$ with $2 \mathrm{mM}$ isopropyl- $\beta$-D-1-thiogalactopyranoside. The $\mathrm{His}_{6} \mathrm{Get} 4 / \mathrm{Get} 5$ complex was then purified using Ni-NTA super flow beads according to the manufacturer's manual under native conditions (QIAGEN) (Supplementary Fig. 4i). Purified $\mathrm{His}_{6} \mathrm{Get} 4 / \mathrm{Get} 5$ was transferred to Hepes buffer (20 mM Hepes/KOH pH 7.4, $2 \mathrm{mM} \mathrm{Mg(OAc})_{2}, 120 \mathrm{mM} \mathrm{KOAc,} 5 \%$ glycerol, $1 \mathrm{mM}$ phenylmethylsulfonyl fluoride (PMSF)) using PD-10 desalting columns (GE Healthcare) and was then employed in the experiments shown in Figs. 4 and 5. Get4/His ${ }_{6}$ Get5 was expressed in E. coli BL21(DE3) from plasmid pETDuet-His ${ }_{6}$ Get5-Get4. Purification of Get $4 / \mathrm{His}_{6} \mathrm{Get} 5$ of was performed using HisTrap HP affinity chromatography (GE Healthcare) followed by size-exclusion chromatography (Superdex 200 10/300 GE Healthcare). Purified Get4/His ${ }_{6}$ Get5 was transferred to $50 \mathrm{mM}$ Tris/ $\mathrm{HCl} \mathrm{pH} \mathrm{7.5,70} \mathrm{mM} \mathrm{NH}_{4} \mathrm{Cl}, 30 \mathrm{mM} \mathrm{KCl}, 7 \mathrm{mM}$ $\mathrm{MgCl}_{2}$ buffer using PD-10 desalting columns (GE Healthcare) prior to fluorescent labeling for anisotropy and FCS measurements shown in Fig. 1. Purified proteins were stored at $-80^{\circ} \mathrm{C}$.

Preparation of $80 \mathrm{~S}$ ribosomes. $80 \mathrm{~S}$ ribosomes and ribosomal subunits were purified from the Saccharomyces cerevisiae strain YAS-2488 as described previously ${ }^{64,65}$. Cells were harvested in mid-log phase and resuspended in $1 \mathrm{ml} \mathrm{g}^{-1}$ of cells in lysis buffer $(20 \mathrm{mM}$ Hepes/KOH pH 7.5, $100 \mathrm{mM} \mathrm{KOAc}, 2.5 \mathrm{mM} \mathrm{Mg}$ $(\mathrm{OAc})_{2}, 1 \mathrm{mg} \mathrm{ml}^{-1}$ heparin sodium salt, $2 \mathrm{mM}$ dithiothreitol (DTT)). Cell pellets frozen in liquid nitrogen were ground using an ultra-centrifugal mill according to the CryoMill protocol (Retsch). The lysate was thawed at $4^{\circ} \mathrm{C}, 100 \mu \mathrm{l}$ DNase and one ethylenediaminetetraacetic acid (EDTA)-free protease inhibitor tablet (Roche) was added and incubated at $4{ }^{\circ} \mathrm{C}$ for $30 \mathrm{~min}$. The thawed lysate was clarified by centrifugation at $25,000 \times g$ in a JLA 16.250 (Beckman Coulter) at $4{ }^{\circ} \mathrm{C}$ for $30 \mathrm{~min}$. The salt concentration of the supernatant was increased to $500 \mathrm{mM} \mathrm{KCl}$ and was then filtered using $1 \mu \mathrm{m}$ glass fiber filters (Pall Corporation). Ribosomes in the supernatant were collected through a $1 \mathrm{M}$ sucrose cushion $(20 \mathrm{mM}$ Hepes/KOH $\mathrm{pH} 7.5,100 \mathrm{mM}$ KOAc, $400 \mathrm{mM} \mathrm{KCl}, 2.5 \mathrm{mM} \mathrm{Mg}(\mathrm{OAc})_{2}, 1 \mathrm{M}$ sucrose, $2 \mathrm{mM}$ DTT) by centrifugation at $235,000 \times g$ at $4^{\circ} \mathrm{C}$ for $2 \mathrm{~h}$ in a Ti45 rotor (Beckman Coulter). Ribosomal pellets were resuspended in resuspension buffer $(20 \mathrm{mM}$
Hepes/KOH pH 7.5, $100 \mathrm{mM} \mathrm{KOAc,} 400 \mathrm{mM} \mathrm{KCl}, 2.5 \mathrm{mM} \mathrm{Mg}(\mathrm{OAc})_{2}, 1 \mathrm{mg} \mathrm{ml}^{-1}$ heparin sodium salt, $2 \mathrm{mM}$ DTT) and were incubated on ice for $15 \mathrm{~min}$. Ribosomes were collected once more through a $1 \mathrm{M}$ sucrose cushion $(20 \mathrm{mM}$ Hepes/ $\mathrm{KOH} \mathrm{pH}$ 7.5, $100 \mathrm{mM}$ KOAc, $400 \mathrm{mM} \mathrm{KCl}, 2.5 \mathrm{mM} \mathrm{Mg}(\mathrm{OAc})_{2}, 1 \mathrm{M}$ sucrose, $2 \mathrm{mM}$ DTT) at $600,000 \times g$ at $4{ }^{\circ} \mathrm{C}$ for $30 \mathrm{~min}$ in a MLA 130 rotor (Beckman Coulter). Ribosomal pellets were then resuspended in storage buffer $(50 \mathrm{mM}$ Hepes/KOH, $100 \mathrm{mM} \mathrm{KCl}$, $250 \mathrm{mM}$ sucrose, $2.5 \mathrm{mM} \mathrm{MgCl}_{2}, 2 \mathrm{mM}$ DTT) at a final concentration of $\sim 10 \mu \mathrm{M}$ and stored at $-80^{\circ} \mathrm{C}$ after being flash frozen in liquid nitrogen.

Fluorescent labeling and fluorescence measurements. Get4/5-Atto655 or Get4/ 5-Atto390 was generated by fluorescence-labeling purified Get $4 / \mathrm{His}_{6} \mathrm{Get} 5$ with a fivefold excess thiol reactive ATTO-655- or ATTO-390-maleimide dye (ATTOTEC) targeting the surface-exposed cysteine of two cysteines present in Get4 $(\mathrm{C} 177,255)$. Note that Get5 does not contain cysteine residues. The reaction was incubated at $20^{\circ} \mathrm{C}$ for $2 \mathrm{~h}$ and was then quenched with $1 \mathrm{mM}$ DTT. Fluorescence labeled protein was purified from free dye using gel filtration chromatography (Superdex 75). Anisotropy measurements were performed at $20^{\circ} \mathrm{C}$ using fluorescence labeled Get4/5-Atto390 in a FluoroMax spectrofluorometer (Horiba Scientific). Samples were excited at $380 \mathrm{~nm}$ and the emission at $470 \mathrm{~nm}$ was measured. Titrations were performed in a $150 \mu \mathrm{l}$ cuvette in buffer $(50 \mathrm{mM}$ Tris/ $\mathrm{HCl} \mathrm{pH} \mathrm{7.5,}$ $70 \mathrm{mM} \mathrm{NH}_{4} \mathrm{Cl}, 30 \mathrm{mM} \mathrm{KCl}, 7 \mathrm{mM} \mathrm{MgCl}_{2}$ ), the concentration of Get4/5-Atto390 was held constant $(20 \mathrm{nM})$ and increasing concentrations of ribosomes were added from a working stock of $1 \mu \mathrm{M}$ to final concentrations as indicated $(20-500 \mathrm{nM})$. Anisotropy $(r)$ was determined from Eq. 1:

$$
r=\frac{I_{V V}-G * I_{V H}}{I_{V V}+2 * G * I_{V H}}
$$

where $I_{V V}$ are the light intensities with excitation and emission polarizers in the vertical direction and $I_{V H}$ uses a vertical excitation polarizer and a horizontal emission polarizer. $G$ is the $\mathrm{G}$ factor measured by Eq. 2 :

$$
G=\frac{I_{H V}}{I_{H H}}
$$

where $I_{H V}$ are the light intensities with a horizontal excitation polarizer and a vertical emission polarizer and $I_{H H}$ are the light intensities with horizontal excitation and emission polarizers.

Anisotropy measurements were plotted as a function of increasing ribosomal concentration and fit with Eq. 3.

$$
A=\frac{A_{\infty} *[R b]}{K_{d}+[R b]}+A_{0}
$$

where $A_{\infty}$ is the anisotropy at saturating concentrations of ribosomes [Rb], $K_{\mathrm{d}}$ is the equilibrium dissociation constant of the complex, and $A_{0}$ is the anisotropy of the free protein.

Fluorescence correlation spectroscopy. Confocal FCS measurements were performed on a PicoQuant MicroTime 200 inverse time-resolved confocal microscope We setup the experiment in a volume of $50 \mu \mathrm{l}$ buffer $(50 \mathrm{mM}$ Tris/ $\mathrm{HCl} \mathrm{pH} \mathrm{7.5,70}$ $\mathrm{mM} \mathrm{NH}_{4} \mathrm{Cl}, 30 \mathrm{mM} \mathrm{KCl}, 7 \mathrm{mM} \mathrm{MgCl}_{2}$ ). The concentration of Get4/5-Atto655 was $5 \mathrm{nM}$. One to two fluorophore molecules were excited using a $635 \mathrm{~nm}$ continuous wave laser at $\sim 80 \mu \mathrm{W}$. This signal was further filtered using two $690 \mathrm{~nm}$ filters and the cross-correlation of the emitted light was collected. Autocorrelation functions were measured using SymPhoTime 64 software where 314 sample points were collected over a $60 \mathrm{sec}$ time period in the presence and absence of $80 \mathrm{~S}$ ribosomes (1 $\mu \mathrm{M}$; diluted from a ca. $10 \mu \mathrm{M}$ stock in ribosomal storage buffer) and additional unlabeled GET proteins (1 $\mu \mathrm{M}$ final concentration). A minimum of six replicates were performed to produce one average trace and subsequently fit with a twodimensional diffusion model ${ }^{66}$.

$$
G(t)=\sum \frac{\rho}{\left[1+\left[\frac{t}{\tau_{\text {Diffi] }}}\right]\right]^{[i]}}+G_{\infty}
$$

$G(t)$ is the autocorrelation function and $G_{\infty}$ is the autocorrelation function at infinity. $T_{\text {Diff }}$ is the diffusion time of the sample and $\rho$ is the contribution of the ith diffusing species. After fitting, $T_{\text {Diff }}$ was determined using Eq. 4 and expressed including the standard error of the parameter obtained from the confidence of the fit. Diffusion coefficients $(D)$ were determined using Eq. 5.

$$
D=\frac{2 * V_{E F F}}{4 * \tau_{D i f f} * K * \pi}
$$

where $V_{E f f}$ is the effective excitation volume and $K$ (kappa) is the length to diameter ratio of the focal volume, both determined from the calibration using free ATTO$655 \mathrm{dye}^{67}$. All measurements were performed at room temperature.

Ribosome-binding assay. Yeast strains were grown to early log phase on YPD, cycloheximide was added to a final concentration of $100 \mu \mathrm{g} \mathrm{ml}^{-1}$ and the cultures were chilled on ice. Cells were harvested by centrifugation at $5000 \times g$. Cell pellets were resuspended in ribosome-binding buffer $(20 \mathrm{mM}$ Hepes/KOH pH 7.4, $2 \mathrm{mM}$ $\mathrm{Mg}(\mathrm{OAc})_{2}, 120 \mathrm{mM} \mathrm{KOAc}, 50 \mu \mathrm{gl}^{-1}$ cycloheximide, $2 \mathrm{mM}$ DTT, $1 \mathrm{mM}$ PMSF 
protease inhibitor mix: $1.25 \mu \mathrm{g} \mathrm{ml}^{-1}$ leupeptin, $0.75 \mu \mathrm{g} \mathrm{ml}^{-1}$ antipain, $0.25 \mu \mathrm{g} \mathrm{ml}^{-1}$ chymostatin, $0.25 \mu \mathrm{g} \mathrm{ml}^{-1}$ elastinal, $5 \mu \mathrm{g} \mathrm{ml}^{-1}$ pepstatin A) and total cell extracts were prepared by the glass beads method ${ }^{68}$. After a clearing spin at $20,000 \times g, 60 \mu \mathrm{l}$ of the total glass beads extract $\left(\mathrm{A}_{260}\right.$ between 80 and $\left.100 \mathrm{mAU}\right)$ was loaded onto a $90 \mu \mathrm{l}$ low salt sucrose cushion (25\% sucrose, $20 \mathrm{mM}$ Hepes/KOH pH 7.4, $120 \mathrm{mM}$ KOAc, $2 \mathrm{mM} \mathrm{Mg}(\mathrm{OAc})_{2}, 2 \mathrm{mM}$ DTT, $1 \mathrm{mM}$ PMSF, protease inhibitor mix) or a 90 $\mu \mathrm{l}$ high-salt sucrose cushion (25\% sucrose, $20 \mathrm{mM}$ Hepes/KOH pH 7.4, $800 \mathrm{mM}$ $\mathrm{KOAc}, 2 \mathrm{mM} \mathrm{Mg}(\mathrm{OAc})_{2}, 2 \mathrm{mM}$ DTT, $1 \mathrm{mM}$ PMSF, protease inhibitor mix). After centrifugation at $400,000 \times \mathrm{g}$ at $4^{\circ} \mathrm{C}$ for $25 \mathrm{~min}$, the cytosolic supernatant was collected and the ribosomal pellet was resuspended in $300 \mu \mathrm{l}$ ribosome-binding buffer. Aliquots of the total cell extract, cytosolic supernatant, and resuspended ribosomes were precipitated by addition of TCA to a final concentration of $5 \%$. TCA pellets were dissolved in SDS sample buffer $(20 \mathrm{mM}$ Tris/ $\mathrm{HCl} \mathrm{pH} \mathrm{6.8,} 1 \%$ SDS, $5 \mathrm{mM}$ EDTA, $0.08 \%$ bromphenolblue, $10 \%$ glycerol,) and were analyzed on $10 \%$ Tris-Tricine gels, followed by immunoblotting. Sse1 or Pgk1 served as cytosolic marker; Rpl35, Rpl31, or Rpl24 served as ribosomal markers.

Ribosome profile analysis. Ribosome profiles were performed with total glass beads extract prepared as described for the ribosome-binding assay. Total glass beads extract corresponding to $10 A_{260}$ units was loaded onto an $11 \mathrm{ml} 15-55 \%$ linear sucrose gradient prepared in ribosome-binding buffer (Gradient Master 108, Biocomp). Samples were then centrifuged for $2.5 \mathrm{~h}$ at $200,000 \times g$ (TH641, Sorvall), and fractionated into $560 \mu \mathrm{l}$ aliquots with a gradient fractionator monitoring $A_{260}$ (Piston Gradient Fractionator, Biocomp). A total corresponding to $5 \%$ of the material loaded onto the gradient, aliquots of fractions 1-8 (560 $\mu \mathrm{l} \mathrm{each),} \mathrm{combined}$ fractions 9-19 $(6.2 \mathrm{ml})$, and the resuspended pellet, which had formed at the bottom of the gradient tube were precipitated by the addition of TCA, and were analyzed on Tris-Tricine gels followed by immunoblotting. Please note, that combined fractions 9-19 correspond to 11 aliquots when compared with the individual fractions $1-8$.

Protein-RNA CRAC. Yeast strains expressing HTP-tagged Get3, Get4, or Sgt2 from their genomic loci or plasmid-derived HTP-tagged Get5 were analyzed by PAR-CRAC ${ }^{33,69}$. Cells were grown exponentially in low uracil media $(10 \mathrm{mg} / \mathrm{l}$ uracil) supplemented with $100 \mu \mathrm{M} 4$-thiouracil before growth for an additional $4 \mathrm{~h}$ in the presence of $1 \mathrm{mM} 4$-thiouracil. 4-thiouridine (4sU)-containing RNAs were crosslinked to associated proteins using $600 \mathrm{~mJ} \mathrm{~cm}^{-2}$ irradiation at $365 \mathrm{~nm}$. Protein-RNA complexes were isolated under native conditions on IgG sepharose and then under denaturing conditions on Ni-NTA. A partial RNase digest was performed using RNace-IT, and co-purified RNAs were $5^{\prime}$ labeled with $\left[{ }^{32} \mathrm{P}\right]$ and were ligated to $3^{\prime}$ and $5^{\prime}$-sequencing adaptors. The $5^{\prime}$ adaptor contained an NNNNNAGC unique molecular identifier sequence (UMI) to allow consolidation of multiple sequencing reads derived from the same RNA template. Complexes were separated by NuPAGE and transferred to a nitrocellulose membrane. RNAs were released from the membrane by Proteinase $\mathrm{K}$ digestion, isolated and reverse transcribed. The cDNA libraries were amplified by PCR (wild type: 35 cycles, Get4HTP: 24 cycles) and subjected to Illumina deep sequencing. Sequencing reads were trimmed and quality controlled using Flexbar ${ }^{70}$, reads shorter than 18 nucleotides were discarded. Identical sequencing reads containing the same UMI were collapsed to a single read. The remaining sequences were mapped to the $S$. cerevisiae genome using Bowtie $2^{71}$. Alignments containing no, or a single, mismatch were allowed and reads were then filtered to retain only those reads containing a single $\mathrm{T}-\mathrm{C}$ mutation induced by the presence of $4 \mathrm{sU}$. For generating heat maps showing the number of reads mapping to different nucleotides on the secondary structure of the $25 \mathrm{~S} \mathrm{rRNA}^{72}$ and the tertiary structure of the ribosome (PDB 4V88), programming scripts in the programming language Python (version 3.5.2) were used.

In vitro transcription and translation. DNA templates for transcription reactions were generated by PCR using pSP65-FLAG-Sec $22+60$ or pSP65-Sec $22+60$ as templates ${ }^{42}$. Reverse primers were designed such that PCR products encoded stop codon-less Sec22, Sec $22+10$, Sec $22+20$, Sec $22+30$, Sec $22+40$, or Sec $22+60$, or the $\mathrm{N}$-terminally FLAG-tagged versions, respectively. In order to generate full-length released Sec22 or FLAG-Sec22 reverse primers containing the UAG stop codon were designed. Transcripts were generated using SP6 polymerase (ThermoFischer Scientific $)^{73}$. Yeast translation extract was prepared from yeast strains as indicated in Results and Figure Legends. Cells harvested from 101 cultures were resuspended in $200 \mathrm{ml}$ sorbitol buffer (1.4 M sorbitol, $50 \mathrm{mM} \mathrm{K}$-phosphate buffer $\mathrm{pH} 7.4,10 \mathrm{mM}$ DTT) containing $2 \mathrm{mg}$ zymolyase $20 \mathrm{~T}$ (Nacalai Tesque Inc.) $\mathrm{g}^{-1}$ cells. After incubation at $30^{\circ} \mathrm{C}$ for $30 \mathrm{~min}$, spheroblasts were collected at $4{ }^{\circ} \mathrm{C}$ and were resuspended in $300 \mathrm{ml}$ sorbitol buffer (YPD medium containing $1 \mathrm{M}$ sorbitol). After incubation at $22^{\circ} \mathrm{C}$ for $90 \mathrm{~min}$, spheroblasts were re-collected, and resuspended in $400 \mathrm{ml}$ sorbitol buffer. The spheroblast suspension was transferred into two $500 \mathrm{ml}$ JA-10 (Beckman Coulter) centrifuge tubes and underlaid with $200 \mathrm{ml}$ of cold sorbitol buffer. After centrifugation at $4000 \times g$ for $7 \mathrm{~min}$ (JA-10), spheroblasts were washed twice with cold sorbitol buffer and resuspended in 5-10 ml lysis buffer (20 $\mathrm{mM}$ Hepes/KOH pH 7.4, $100 \mathrm{mM} \mathrm{KOAc,} 2 \mathrm{mM} \mathrm{MgOAc} 2,2 \mathrm{mM}$ DTT, $0.5 \mathrm{mM}$ PMSF, $1 \times$ protease inhibitor mix). The spheroblast suspension was transferred into a $40 \mathrm{ml}$ dounce homogenizer (Kontes Glass Co.) and spheroblasts were disrupted on ice by douncing with a type $\mathrm{B}$ pestle. The resulting extract was centrifuged at
$27,000 \times g$ in a SS34 rotor (Piramoon Technologies Inc.) for $18 \mathrm{~min}$ at $4{ }^{\circ} \mathrm{C}$. The supernatant was collected and centrifuged at $100,000 \times g$ in a 70.1 Ti rotor (Beckman Coulter) for $35 \mathrm{~min}$ at $4^{\circ} \mathrm{C}$. The supernatant was loaded onto a Superdex-G25 gel filtration column equilibrated with gel filtration buffer $(20 \mathrm{mM} H e p e s / \mathrm{KOH} \mathrm{pH}$ 7.4, $100 \mathrm{mM} \mathrm{KOAc,} 2 \mathrm{mM} \mathrm{MgOAc}_{2}, 2 \mathrm{mM}$ DTT, $0.5 \mathrm{mM}$ PMSF, 20\% glycerol) at flow rate of $1.5 \mathrm{ml} \mathrm{min}^{-1}$. Peak fractions with the highest $\mathrm{A}_{260}$ were pooled, were supplemented with $1 \mathrm{mM} \mathrm{CaCl}_{2}$, were treated with $300 \mathrm{U} \mathrm{ml}^{-1}$ micrococcal nuclease (Roche) for $15 \mathrm{~min}$ at $20^{\circ} \mathrm{C}$ followed by addition of $2 \mathrm{mM}$ EGTA, and aliquots of the resulting yeast translation extract, were stored at $-80^{\circ} \mathrm{C}^{74,75}$. RNCs were generated via translation reactions primed with the stop codon-less transcripts at $20^{\circ} \mathrm{C}$ for $80 \mathrm{~min}^{42,74}$. RNCs for chemical crosslinking were generated in the presence of $\left[{ }^{35} \mathrm{~S}\right]$-methionine (Hartmann-Analytic). RNCs were isolated via centrifugation at $400,000 \times g$ for 20 min as described ${ }^{42}$. Released Sec 22 or FLAG-Sec 22 chains for pull-down assays were labeled with $\left[{ }^{35} \mathrm{~S}\right]$-methionine and were isolated away from ribosomes via centrifugation at $400,000 \times g$ for $20 \mathrm{~min}$. As indicated purified $\mathrm{His}_{6} \mathrm{Get} 4 / \mathrm{Get} 5$ was added to in vitro translation reactions to a final concentration of $2 \mu \mathrm{M}$

Chemical crosslinking of $\mathrm{His}_{6}$ Get5 and Ni-NTA purification under denaturing conditions. Total cell extracts were prepared according to the CryoMill protocol (Retsch $\left.{ }^{\circledast}\right)$. CryoMill powder was dissolved in lysis buffer $(20 \mathrm{mM} H e p e s / \mathrm{KOH} \mathrm{pH}$ 7.4, $2 \mathrm{mM} \mathrm{Mg}(\mathrm{OAc})_{2}, 120 \mathrm{mM} \mathrm{KOAc}, 50 \mu \mathrm{g} \mathrm{ml}^{-1}$ cycloheximide, $2 \mathrm{mM}$ DTT, 1 $\mathrm{mM}$ PMSF, protease inhibitor mix) and ribosomes were isolated by a centrifugation step at $400,000 \times g$ at $4{ }^{\circ} \mathrm{C}$ for $25 \mathrm{~min}$. $\mathrm{BS}^{3}$ (bis-(sulfosuccinimidyl)-suberate, spacer length $1.14 \mathrm{~nm}$, Thermo Scientific) was added to resuspended ribosomes to a final concentration of $150 \mu \mathrm{M}$, and samples were incubated on ice for $15 \mathrm{~min}$. Crosslinking was then quenched by the addition of Tris base (tris(hydroxymethyl) aminomethane) to a final concentration of $50 \mathrm{mM}$. Crosslinked ribosomes were reisolated by ultracentrifugation as described above and ribosomal pellets were resuspended in denaturing binding buffer $(8 \mathrm{M}$ urea, $0.1 \mathrm{M}$ sodium phosphate buffer, $0.01 \mathrm{M}$ Tris/HCl, $\mathrm{pH}$ 8.0). The material was applied to Ni-NTA super flow beads according to the manufacturer's manual under denaturing conditions (QIAGEN) using denaturing wash buffer ( $8 \mathrm{M}$ urea, $0.1 \mathrm{M}$ sodium phosphate, 0.01 $\mathrm{M}$ Tris/ $\mathrm{HCl} \mathrm{pH}$ 6.3) and, for the elution of $\mathrm{His}_{6} \mathrm{Get} 5$ and crosslinked proteins denaturing low- $\mathrm{pH}$ elution buffer $(8 \mathrm{M}$ urea, $0.1 \mathrm{M}$ sodium phosphate, $0.01 \mathrm{M}$ Tris/ $\mathrm{HCl} \mathrm{pH} \mathrm{4.5).} \mathrm{TCA} \mathrm{was} \mathrm{added} \mathrm{to} \mathrm{the} \mathrm{eluates} \mathrm{to} \mathrm{a} \mathrm{final} \mathrm{concentration} \mathrm{of} 5 \%$ and protein pellets were dissolved in SDS sample buffer and analyzed on Tris-Tricine gels followed by immunoblotting or analysis by mass spectrometry (Fig. 2a).

Mass spectrometry. Crosslinked samples were diluted in NuPAGE SDS sample buffer and were run on 4-12\% NuPAGE gels (Supplementary Fig. 2c). Each sample was cut into 11 slices and was digested with trypsin. Following digestion, two technical replicates of LC-MS-MS were performed. All peptides were screened against a UniProt yeast database and further analyzed via the Scaffold 4.9.0. software (Supplementary Dataset 1 and Source data file). Selection of ribosomal crosslinking partners of $\mathrm{His}_{6}$ Get5 (Table 1) is described in Results. Yeast possesses two paralogues (designated a and b) of many ribosomal protein genes. The corresponding proteins often differ in only a few residues. If the mass spec results did not allow to distinguish between $\mathrm{a}$ and $\mathrm{b}$ forms, the data were included into the same cluster.

\section{Chemical crosslinking of RNCs and denaturing immunoprecipitation of} crosslink products. RNCs prepared by in vitro translation (see above) were separated from cytosolic components and released nascent chains by centrifugation through a sucrose cushion at $400,000 \times g$ for $20 \mathrm{~min}$. For that purpose, $60 \mu \mathrm{l}$ translation reaction were loaded onto a $90 \mu \mathrm{l}$ cushion (25\% sucrose in resuspension buffer: $20 \mathrm{mM}$ Hepes/KOH pH 7.4, $2 \mathrm{mM} \mathrm{Mg}(\mathrm{OAc})_{2}, 120 \mathrm{mM} \mathrm{KOAc,} 1 \mathrm{mM}$ PMSF, protease inhibitor mix). After resuspension of RNCs in resuspension buffer crosslinking was performed by the addition of $\mathrm{BS}^{3}$ to a final concentration of 400 $\mu \mathrm{M}$ for $20 \mathrm{~min}$ on ice. Crosslinking reactions were then quenched by the addition of Tris base to a final concentration of $50 \mathrm{mM}$. Crosslinked protein samples were precipitated by the addition of TCA (final concentration 5\%). Pellets were collected by centrifugation and were dissolved in dissociation buffer $(200 \mathrm{mM}$ Tris/ $\mathrm{HCl} \mathrm{pH}$ 7.5, 4\% SDS; $10 \mathrm{mM}$ EDTA, $100 \mu \mathrm{g} \mathrm{ml}^{-1} \mathrm{BSA}$, protease inhibitor mix, $1 \mathrm{mM}$ PMSF). The resulting denatured protein samples were used to identify crosslink products between SRP, Get4/5, or Sgt 2 and nascent chains via affinity purification. For this purpose, protein A sepharose beads (GE Healthcare) were pre-coated with antibodies (Eurogentec) directed against Srp54, Get4, Get5, or Sgt2 and subsequently the denatured crosslinked material was allowed to bind to the beads ${ }^{42}$ Crosslink products bound to protein A sepharose beads were released by addition of SDS sample buffer at $95^{\circ} \mathrm{C}$ for $10 \mathrm{~min}$. Samples were analyzed on $10 \%$ TrisTricine gels followed by autoradiography.

FLAG-tag pull-down reactions. FLAG-tag pull-down experiments were performed as described ${ }^{20}$. RNCs were separated from cytosolic components by centrifugation through a sucrose cushion as described above, were resuspended in resuspension buffer and were then added to $20 \mu \mathrm{l}$ of pre-washed Anti-FLAG ${ }^{\oplus}$ M2 affinity beads (FLAG-beads, Sigma) resuspended in $600 \mu \mathrm{l}$ resuspension buffer. Non-tagged and N-terminally FLAG-tagged RNCs were analyzed in parallel in 
each experiment to determine background binding (Supplementary Fig. $4 \mathrm{~b}$ and Supplementary Fig. $5 \mathrm{f}$ ). Material bound to FLAG-beads was separated on $10 \%$ TrisTricine gels, transferred to nitrocellulose membranes, and was analyzed via immunoblotting. In case FLAG-tag pull-down experiments were performed with released Sec22 chains, the samples were diluted 1:7 with resuspension buffer and were then subjected to FLAG-tag pull-down reactions. After the binding reaction, FLAG-beads were washed with resuspension buffer and bound material was eluted by incubation with SDS sample buffer at $30^{\circ} \mathrm{C}$ for $20 \mathrm{~min}$. Subsequently, SDS sample buffer was removed from the beads, cleared by centrifugation at $5000 \times g$ for $30 \mathrm{sec}$, transferred into a fresh $1.5 \mathrm{ml}$ tube, and boiled at $95^{\circ} \mathrm{C}$ for $5 \mathrm{~min}$.

Quantification of ribosome-bound Sgt2 in FLAG-tag pull-down reactions. The relative occupancy of RNCs with Sgt2 was determined by quantitative analysis of experimental data as shown in Fig. 4f. For each replicate, the amount of Sgt2 and RNCs (Rps9) was determined by densitometry. As a first step, Sgt2 values were normalized for the amount of RNCs (ratio: Sgt2/Rps9). Normalized Sgt2 values obtained for the $\Delta s r p 54$ samples were set to 1 . The amount of Sgt2 bound to wildtype RNCs-Sec22+60 without addition of purified $\mathrm{His}_{6}$ Get $4 / \mathrm{Get} 5$ was below the detection limit of ImageJ.

Quantification of the capture of released Sec22 by Sgt2. The relative efficiency with which Sgt2 was bound to released Sec22 was determined by quantitative analysis of data as shown in Fig. 5e. For each replicate, the relative amount of Sgt2 was determined by densitometry of immunoblots. In parallel, the amount of $\left[{ }^{35} \mathrm{~S}\right]-$ labeled Sec22 was determined by autoradiography. Sgt2 values were normalized for the amount of $\left[{ }^{35} \mathrm{~S}\right]$-labeled Sec22 (ratio: Sgt2/Sec22) and normalized Sgt2 values in the $\Delta$ get 3 samples were set to 1 .

Statistics and reproducibility. The following experiments were repeated independently with similar results and represent biological replicates. Figure la: $n=3$. Figure 1c: $n=3$ for each concentration, except for the measurements at $0.1 \mu \mathrm{M} 80 \mathrm{~S}$ ribosome concentration, which was $n=2$. Figure $1 \mathrm{~d}: n=6$. Figure $2 \mathrm{~b}-\mathrm{d}: n=2$. Figure $3 \mathrm{~b}: n=2$. Figure $4 \mathrm{~b}: n=2$. Figure $4 \mathrm{c}: n=3$. Figure. $4 \mathrm{~d}$, e: $n=2$. Figure. $4 \mathrm{f}$, g: wild type and $\Delta \operatorname{srp} 54 n=5$, wild type $+\mathrm{His}_{6} \mathrm{Get}_{4} / \mathrm{Get} 5$ and $\Delta \operatorname{srp} 54+\mathrm{His}_{6} \mathrm{Get}_{4} /$ Get5 $n=2$. Figure 5a: $n=1$. Figure. 5b-d: $n=2$. Figure 5e: $n=3$. Figure 6a: $n=3$. Figure. $6 \mathrm{c}-\mathrm{f}: n=2$. Supplementary Fig. 1: $n=2$. Supplementary Fig. 2a, b: $n=2$. Supplementary Fig. 2c: $n=2$ (technical replicates of LC-MS). Supplementary Fig. 3a-e: $n=1$. Supplementary Fig. 3f: $n=2$. Supplementary Fig. $4 \mathrm{c}, 4 \mathrm{~d}$, and $4 \mathrm{f}$ : $n=1$. Supplementary Fig. 4 e, j, and $4 \mathrm{k}: n=2$. Supplementary Fig. $5 \mathrm{~b}-\mathrm{d}, 5 \mathrm{~g}$, and 5h: $n=2$.

Miscellaneous. Immunoblots were developed using an ECL camera (Fusion Pulse 6, Vilber). Autoradiographs were analyzed and quantified with a Typhoon 9410 (GE Healthcare Life Sciences). Quantitative analysis of immunoblots was performed using ImageJ 1.52a (National Institutes of Health, USA). Statistical analysis was performed with GraphPad Prism 6.07. Models of ribosomal particles were generated with PyMOL 2.2.0. Polyclonal antibodies directed against Srp54 42 (1:4000), Sgt2 24 (1:5000), Get4 ${ }^{24}$ (1:5000), Get524 (1:5000), Get324 (1:5000), Rpl4 ${ }^{24}$ (1:10,000), Rpl2442 (1:10,000), Rpl35 $5^{60}$ (1:10,000), Rpl31 ${ }^{60}$ (1:5000), Rpl26 60 (1:2000), Rps9 $9^{42}$ (1:5000), Pgk1 $1^{75}$ (1:2000), Kar2 ${ }^{76}(1: 2000), \operatorname{Sse}^{42}(1: 20,000)$ (Eurogentec, Rospert lab antibody collection), and PAP (Sigma ID P1291, 1:5000) were raised in rabbit. $\alpha$-FLAG (Sigma F3165, 1:2000) and $\alpha$-His (Qiagen 34660, 1:2000) are monoclonal antibodies. Horseradish peroxidase-conjugated protein A (ThermoFischer Scientific 101023, 1:5000) was employed as a secondary reagent to detect primary antibodies. Dilutions employed for immunoblotting experiments are given in brackets. References, which include validation data of the different antibodies are indicated.

Reporting summary. Further information on research design is available in the Nature Research Reporting Summary linked to this article.

\section{Data availability}

The CRAC data sets of Get4-HTP and the wild type yeast control are deposited in the Gene Expression Omnibus database under the accession code GSE151664. Other data that support the findings of this study and biological materials are available from the corresponding authors on reasonable request. Source data are provided with this paper.

Received: 10 July 2020; Accepted: 5 January 2021;

Published online: 04 February 2021

\section{References}

1. Hegde, R. S. \& Keenan, R. J. Tail-anchored membrane protein insertion into the endoplasmic reticulum. Nat. Rev. Mol. Cell Biol. 12, 787-798 (2011).
2. Shao, S. \& Hegde, R. S. Membrane protein insertion at the endoplasmic reticulum. Annu. Rev. Cell Dev. Biol. 27, 25-56 (2011).

3. Nyathi, Y., Wilkinson, B. M. \& Pool, M. R. Co-translational targeting and translocation of proteins to the endoplasmic reticulum. Biochim. Biophys. Acta 1833, 2392-2402 (2013)

4. Voorhees, R. M. \& Hegde, R. S. Structures of the scanning and engaged states of the mammalian SRP-ribosome complex. Elife 4, e07975 (2015).

5. Aviram, N. \& Schuldiner, M. Targeting and translocation of proteins to the endoplasmic reticulum at a glance. J. Cell Sci. 130, 4079-4085 (2017).

6. Stefanovic, S. \& Hegde, R. S. Identification of a targeting factor for posttranslational membrane protein insertion into the ER. Cell 128, 1147-1159 (2007).

7. Favaloro, V., Spasic, M., Schwappach, B. \& Dobberstein, B. Distinct targeting pathways for the membrane insertion of tail-anchored (TA) proteins. J. Cell Sci. 121, 1832-1840 (2008).

8. Schuldiner, M. et al. The GET complex mediates insertion of tail-anchored proteins into the ER membrane. Cell 134, 634-645 (2008).

9. Jonikas, M. C. et al. Comprehensive characterization of genes required for protein folding in the endoplasmic reticulum. Science 323, 1693-1697 (2009).

10. Wang, F., Brown, E. C., Mak, G., Zhuang, J. \& Denic, V. A chaperone cascade sorts proteins for posttranslational membrane insertion into the endoplasmic reticulum. Mol. Cell 40, 159-171 (2010).

11. Chio, U. S., Cho, H. \& Shan, S. O. Mechanisms of tail-anchored membrane protein targeting and insertion. Annu. Rev. Cell Dev. Biol. 33, 417-438 (2017).

12. Chartron, J. W., Gonzalez, G. M. \& Clemons, W. M. Jr. A structural model of the Sgt2 protein and its interactions with chaperones and the Get $4 / \mathrm{Get} 5$ complex. J. Biol. Chem. 286, 34325-34334 (2011)

13. Gristick, H. B. et al. Mechanism of assembly of a substrate-transfer complex during tail-anchored protein targeting. J. Biol. Chem. 290, 30006-17 (2015).

14. Casson, J., McKenna, M. \& High, S. On the road to nowhere: cross-talk between post-translational protein targeting and cytosolic quality control. Biochem. Soc. Trans. 44, 796-801 (2016).

15. Mateja, A. \& Keenan, R. J. A structural perspective on tail-anchored protein biogenesis by the GET pathway. Curr. Opin. Struct. Biol. 51, 195-202 (2018).

16. Borgese, N., Coy-Vergara, J., Colombo, S. F. \& Schwappach, B. The ways of tails: the GET pathway and more. Protein J. 38, 289-305 (2019).

17. McDowell, M. A. et al. Structural basis of tail-anchored membrane protein biogenesis by the GET insertase complex. Mol. Cell 290, 30006-17 (2020).

18. Berndt, U., Oellerer, S., Zhang, Y., Johnson, A. E. \& Rospert, S. A signalanchor sequence stimulates signal recognition particle binding to ribosomes from inside the exit tunnel. Proc. Natl Acad. Sci. USA 106, 1398-1403 (2009).

19. Mariappan, M. et al. A ribosome-associating factor chaperones tail-anchored membrane proteins. Nature 466, 1120-1124 (2010).

20. Zhang, Y. et al. NAC functions as a modulator of SRP during the early steps of protein targeting to the ER. Mol. Biol. Cell 23, 3027-3040 (2012).

21. Holtkamp, W. et al. Dynamic switch of the signal recognition particle from scanning to targeting. Nat. Struct. Mol. Biol. 19, 1332-1337 (2012).

22. Mercier, E., Holtkamp, W., Rodnina, M. V. \& Wintermeyer, W. Signal recognition particle binds to translating ribosomes before emergence of a signal anchor sequence. Nucleic Acids Res. 45, 11858-11866 (2017).

23. Fleischer, T. C., Weaver, C. M., McAfee, K. J., Jennings, J. L. \& Link, A. J. Systematic identification and functional screens of uncharacterized proteins associated with eukaryotic ribosomal complexes. Genes Dev. 20, 1294-1307 (2006).

24. Zhang, Y. et al. Cotranslational intersection between the SRP and GET targeting pathways to the ER of Saccharomyces cerevisiae. Mol. Cell Biol. 36, 2374-2383 (2016).

25. Chiabudini, M., Conz, C., Reckmann, F. \& Rospert, S. RAC/Ssb is required for translational repression induced by polylysine segments within nascent chains. Mol. Cell Biol. 32, 4769-4779 (2012).

26. Morgan, D. G. et al. A comparison of the yeast and rabbit 80 S ribosome reveals the topology of the nascent chain exit tunnel, inter-subunit bridges and mammalian rRNA expansion segments. J. Mol. Biol. 301, 301-321 (2000).

27. Garcia De La Torre, J., Huertas, M. L. \& Carrasco, B. Calculation of hydrodynamic properties of globular proteins from their atomic-level structure. Biophys. J. 78, 719-730 (2000).

28. Chartron, J. W., Suloway, C. J., Zaslaver, M. \& Clemons, W. M. Jr. Structural characterization of the Get $4 /$ Get 5 complex and its interaction with Get3. Proc. Natl. Acad. Sci. USA 107, 12127-12132 (2010).

29. Nieuwenhuysen, P. \& Clauwaert, J. Physicochemical characterization of ribosomal particles from the eukaryote Artemia. J. Biol. Chem. 256, 9626-9632 (1981).

30. Müller, L. et al. Evolutionary gain of function for the ER membrane protein Sec62 from yeast to humans. Mol. Biol. Cell 21, 691-703 (2010).

31. Müller, S. A. et al. Optimization of parameters for coverage of low molecular weight proteins. Anal. Bioanal. Chem. 398, 2867-2881 (2010). 
32. Bohnsack, M. T., Tollervey, D. \& Granneman, S. Identification of RNA helicase target sites by UV cross-linking and analysis of cDNA. Methods Enzymol. 511, 275-288 (2012).

33. Brüning, L. et al. RNA helicases mediate structural transitions and compositional changes in pre-ribosomal complexes. Nat. Commun. 9, 5383 (2018).

34. Memet, I., Doebele, C., Sloan, K. E. \& Bohnsack, M. T. The G-patch protein NF-kappaB-repressing factor mediates the recruitment of the exonuclease XRN2 and activation of the RNA helicase DHX15 in human ribosome biogenesis. Nucleic Acids Res. 45, 5359-5374 (2017).

35. Pool, M. R., Stumm, J., Fulga, T. A., Sinning, I. \& Dobberstein, B. Distinct modes of signal recognition particle interaction with the ribosome. Science 297, 1345-1348 (2002).

36. Halic, M. et al. Structure of the signal recognition particle interacting with the elongation-arrested ribosome. Nature 427, 808-814 (2004).

37. Wegrzyn, R. D. et al. A conserved motif is prerequisite for the interaction of NAC with ribosomal protein L23 and nascent chains. J. Biol. Chem. 281, 2847-2857 (2006).

38. Gamerdinger, M. et al. Early scanning of nascent polypeptides inside the ribosomal tunnel by NAC. Mol. Cell 75, 996-1006.e8 (2019).

39. Nyathi, Y. \& Pool, M. R. Analysis of the interplay of protein biogenesis factors at the ribosome exit site reveals new role for NAC. J. Cell Biol. 210, 287-301 (2015).

40. Becker, T. et al. Structure of monomeric yeast and mammalian Sec61 complexes interacting with the translating ribosome. Science 326, 1369-1373 (2009).

41. Voorhees, R. M., Fernandez, I. S., Scheres, S. H. \& Hegde, R. S. Structure of the mammalian ribosome-Sec61 complex to 3.4 A resolution. Cell 157, 1632-1643 (2014).

42. Raue, U., Oellerer, S. \& Rospert, S. Association of protein biogenesis factors at the yeast ribosomal tunnel exit is affected by the translational status and nascent polypeptide sequence. J. Biol. Chem. 282, 7809-7816 (2007).

43. Zhang, Y., Wölfle, T. \& Rospert, S. Interaction of nascent chains with the ribosomal tunnel proteins Rpl4, Rpl17, and Rpl39 of Saccharomyces cerevisiae. J. Biol. Chem. 288, 33697-33707 (2013).

44. Chartron, J. W., VanderVelde, D. G. \& Clemons, W. M. Jr. Structures of the Sgt2/SGTA dimerization domain with the Get5/UBL4A UBL domain reveal an interaction that forms a conserved dynamic interface. Cell Rep. 2, 1620-1632 (2012).

45. Cho, H. \& Shan, S. O. Substrate relay in an Hsp70-cochaperone cascade safeguards tail-anchored membrane protein targeting. EMBO J. 37, e99264 (2018).

46. Chartron, J. W., VanderVelde, D. G., Rao, M. \& Clemons, W. M. Jr. Get5 carboxyl-terminal domain is a novel dimerization motif that tethers an extended Get4/Get5 complex. J. Biol. Chem. 287, 8310-8317 (2012).

47. Cherry, J. M. et al. Saccharomyces Genome Database: the genomics resource of budding yeast. Nucleic Acids Res. 40, D700-D705 (2012).

48. Ogg, S. C. \& Walter, P. SRP samples nascent chains for the presence of signal sequences by interacting with ribosomes at a discrete step during translation elongation. Cell 81, 1075-1084 (1995).

49. Flanagan, J. J. et al. Signal recognition particle binds to ribosome-bound signal sequences with fluorescence-detected subnanomolar affinity that does not diminish as the nascent chain lengthens. J. Biol. Chem. 278, 18628-18637 (2003).

50. Lee, J. H. et al. Sequential activation of human signal recognition particle by the ribosome and signal sequence drives efficient protein targeting. Proc. Natl. Acad. Sci. USA 115, E5487-E5496 (2018).

51. Liou, S. T., Cheng, M. Y. \& Wang, C. SGT2 and MDY2 interact with molecular chaperone YDJ1 in Saccharomyces cerevisiae. Cell Stress Chaperones 12, 59-70 (2007).

52. Kohl, C. et al. Cooperative and independent activities of Sgt 2 and Get5 in the targeting of tail-anchored proteins. Biol. Chem. 392, 601-608 (2011).

53. Mock, J. Y. et al. Bag6 complex contains a minimal tail-anchor-targeting module and a mock BAG domain. Proc. Natl. Acad. Sci. USA 112, 106-11 (2015).

54. Shao, S., Rodrigo-Brenni, M. C., Kivlen, M. H. \& Hegde, R. S. Mechanistic basis for a molecular triage reaction. Science 355, 298-302 (2017).

55. Wang, Q. et al. A ubiquitin ligase-associated chaperone holdase maintains polypeptides in soluble states for proteasome degradation. Mol. Cell 42, 758-770 (2011).

56. Rodrigo-Brenni, M. C., Gutierrez, E. \& Hegde, R. S. Cytosolic quality control of mislocalized proteins requires RNF126 recruitment to Bag6. Mol. Cell 55, 227-237 (2014).

57. Leznicki, P. \& High, S. SGTA associates with nascent membrane protein precursors. EMBO Rep. 21, e48835 (2020).

58. Brachmann, C. B. et al. Designer deletion strains derived from Saccharomyces cerevisiae S288C: a useful set of strains and plasmids for PCR-mediated gene disruption and other applications. Yeast 14, 115-132 (1998).
59. Heitman, J., Movva, N. R., Hiestand, P. C. \& Hall, M. N. FK 506-binding protein proline rotamase is a target for the immunosuppressive agent FK 506 in Saccharomyces cerevisiae. Proc. Natl Acad. Sci. USA 88, 1948-1952 (1991).

60. Gumiero, A. et al. Interaction of the cotranslational Hsp70 Ssb with ribosomal proteins and rRNA depends on its lid domain. Nat. Commun. 7, 1-12 (2016).

61. Gietz, R. D. \& Sugino, A. New yeast-Escherichia coli shuttle vectors constructed with in vitro mutagenized yeast genes lacking six-base pair restriction sites. Gene 74, 527-534 (1988).

62. Sikorski, R. S. \& Hieter, P. 1, yeast \& vectors, y. A system of shuttle vectors and yeast host strains designed for efficient manipulation of DNA in Saccharomyces cerevisiae. Genetics 122, 19-27 (1989).

63. Martin, R. et al. A pre-ribosomal RNA interaction network involving snoRNAs and the Rok1 helicase. RNA 20, 1173-1182 (2014).

64. Algire, M. A. et al. Development and characterization of a reconstituted yeast translation initiation system. RNA 8, 382-397 (2002).

65. Acker, M. G., Kolitz, S. E., Mitchell, S. F., Nanda, J. S. \& Lorsch, J. R. Reconstitution of yeast translation initiation. Methods Enzymol. 430, 111-145 (2007).

66. Krichevsky, O. \& Bonnet, G. Fluorescence correlation spectroscopy: the technique and its applications. Rep. Prog. Phys. 65, 251-297 (2002).

67. Müller, C. B. et al. Precise measurement of diffusion by multi-color dual-focus fluorescence correlation spectroscopy. EPL 83, 46001 (2008).

68. Ashe, M. P., De Long, S. K. \& Sachs, A. B. Glucose depletion rapidly inhibits translation initiation in yeast. Mol. Biol. Cell 11, 833-848 (2000).

69. Baejen, C. et al. Transcriptome maps of mRNP biogenesis factors define premRNA recognition. Mol. Cell 55, 745-757 (2014).

70. Dodt, M., Roehr, J. T., Ahmed, R. \& Dieterich, C. FLEXBAR-flexible barcode and adapter processing for next-generation sequencing platforms. Biol. (Basel) 1, 895-905 (2012)

71. Langmead, B. \& Salzberg, S. L. Fast gapped-read alignment with Bowtie 2. Nat. Methods 9, 357-359 (2012).

72. Petrov, A. S. et al. Secondary structures of rRNAs from all three domains of life. PLoS ONE 9, e88222 (2014).

73. Fünfschilling, U. \& Rospert, S. Nascent Polypeptide-associated Complex Stimulates Protein Import into Yeast Mitochondria. Mol. Biol. Cell. 10, 3289-3299 (1999).

74. Garcia, P. D., Hansen, W. \& Walter, P. In vitro protein translocation across microsomal membranes of Saccharomyces cerevisiae. Methods Enzymol. 194, 675-682 (1991).

75. Zhang, Y. et al. The ribosome-associated complex RAC serves in a relay that directs nascent chains to Ssb. Nat. Commun. 11, 1504 (2020).

76. Frey, S., Pool, M. \& Seedorf, M. Scp160p, an RNA-binding, polysomeassociated protein, localizes to the endoplasmic reticulum of Saccharomyces cerevisiae in a microtubule-dependent manner. J. Biol. Chem. 276 15905-15912 (2001).

77. Ban, N. et al. A new system for naming ribosomal proteins. Curr. Opin. Struct Biol. 24, 165-169 (2014).

78. Ben-Shem, A. et al. The structure of the eukaryotic ribosome at $3.0 \mathrm{~A}$ resolution. Science 334, 1524-1529 (2011).

\section{Acknowledgements}

This work was supported by the Deutsche Forschungsgemeinschaft (DFG, German Research Foundation) through Project-ID 403222702-SFB 1381, TP B08 (to S.R.), RO 1028/5-1 (to S.R.), SFB 1190 (to K.E.B., B.S. and M.V.R.), and through Germany's Excellence Strategy-EXC 2067/1- 390729940 (to B.S. and M.V.R.). We thank Ákos Farkas for valuable comments and Tessa Hübner for expert technical assistance. The UMG Proteomics Service Facility (Henning Urlaub and Christof Lenz, SFB 1190) are acknowledged for expert help and service.

\section{Author contributions}

Y.Z., E.D., K.E.B., N.R., S.G., M.V.R., B.S., and S.R. designed the experiments and analyzed the data. Y.Z., E.D., K.E.B., M.W., N.R., S.G., P.H., and T.W. performed experiments. Y.Z., E.D., K.E.B., M.V.R., B.S., and S.R. wrote the manuscript. All authors commented on the manuscript.

\section{Funding}

Open Access funding enabled and organized by Projekt DEAL.

\section{Competing interests}

The authors declare no competing interests. 


\section{Additional information}

Supplementary information The online version contains supplementary material available at https://doi.org/10.1038/s41467-021-20981-3.

Correspondence and requests for materials should be addressed to B.S. or S.R.

Peer review information Nature Communications thanks Clémentine Delan-Forino and other, anonymous, reviewers for their contributions to the peer review of this work. Peer review reports are available.

Reprints and permission information is available at http://www.nature.com/reprints

Publisher's note Springer Nature remains neutral with regard to jurisdictional claims in published maps and institutional affiliations. (c) (1) Open Access This article is licensed under a Creative Commons Attribution 4.0 International License, which permits use, sharing, adaptation, distribution and reproduction in any medium or format, as long as you give appropriate credit to the original author(s) and the source, provide a link to the Creative Commons license, and indicate if changes were made. The images or other third party material in this article are included in the article's Creative Commons license, unless indicated otherwise in a credit line to the material. If material is not included in the article's Creative Commons license and your intended use is not permitted by statutory regulation or exceeds the permitted use, you will need to obtain permission directly from the copyright holder. To view a copy of this license, visit http://creativecommons.org/ licenses/by/4.0/.

(C) The Author(s) 2021 